\title{
Existence of solutions to fourth-order differential equations with deviating arguments
}

\author{
Mostepha Naceri', Ravi P Agarwal2,3* Erbil Çetin ${ }^{2,4}$ and El Haffaf Amir ${ }^{5}$
}

"Correspondence: agarwal@tamuk.edu

${ }^{2}$ Department of Mathematics, Texas A\&M University-Kingsville, 700

University Blvd., Kingsville, TX 78363-8202, USA

${ }^{3}$ Department of Mathematics, Faculty of Science, King Abdulaziz University, P.O. Box 80203, Jeddah, 21589, Saudi Arabia Full list of author information is available at the end of the article

\begin{abstract}
In this paper, we consider fourth-order differential equations on a half-line with deviating arguments of the form $u^{(4)}(t)+q(t) f\left(t,[u(t)],\left[u^{\prime}(t)\right],\left[u^{\prime \prime}(t)\right], u^{\prime \prime \prime}(t)\right)=0$, $0<t<+\infty$, with the boundary conditions $u(0)=A, u^{\prime}(0)=B, u^{\prime \prime}(t)-a u^{\prime \prime \prime}(t)=\theta(t)$, $-\tau \leq t \leq 0 ; u^{\prime \prime \prime}(+\infty)=C$. We present sufficient conditions for the existence of a solution between a pair of lower and upper solutions by using Schäuder's fixed point theorem. Also, we establish the existence of three solutions between two pairs of lower and upper solutions by using topological degree theory. An important feature of our existence criteria is that the obtained solutions may be unbounded. We illustrate the importance of our results through two simple examples.
\end{abstract}

MSC: $34 \mathrm{~B} 15 ; 34 \mathrm{~B} 40$

Keywords: fourth-order; boundary value problem; half-line; upper solution; lower solution

\section{Introduction}

In recent years considerable attention has been focused on the existence of solutions to boundary value problems involving differential equations with deviating arguments (DEDA) [1-16]. While most of these works deal with problems on finite intervals and the literature is satisfactory, study of infinite interval problems has been just initiated in $[2$, 13-17]. This study compare to boundary value problems for second and higher order ordinary differential equations over infinite intervals (and their wide variety of applications to real world problems) [18-24] is far from complete, and needs attention. To fill some of this gap, in this paper we shall provide existence criteria for fourth-order differential equations with deviating arguments of the form

$$
u^{(4)}(t)+q(t) f\left(t,[u(t)],\left[u^{\prime}(t)\right],\left[u^{\prime \prime}(t)\right], u^{\prime \prime \prime}(t)\right)=0, \quad 0<t<+\infty,
$$

where

$$
\begin{aligned}
& {[u(t)]=\left(u(t), u\left(t-\tau_{0,1}(t)\right), \ldots, u\left(t-\tau_{0, n}(t)\right)\right),} \\
& {\left[u^{\prime}(t)\right]=\left(u^{\prime}(t), u^{\prime}\left(t-\tau_{1,1}(t)\right), \ldots, u^{\prime}\left(t-\tau_{1, n}(t)\right)\right),} \\
& {\left[u^{\prime \prime}(t)\right]=\left(u^{\prime \prime}(t), u^{\prime \prime}\left(t-\tau_{2,1}(t)\right), \ldots, u^{\prime \prime}\left(t-\tau_{2, n}(t)\right)\right),}
\end{aligned}
$$

(c) 2015 Naceri et al. This article is distributed under the terms of the Creative Commons Attribution 4.0 International License (http://creativecommons.org/licenses/by/4.0/), which permits unrestricted use, distribution, and reproduction in any medium, provided you give appropriate credit to the original author(s) and the source, provide a link to the Creative Commons license, and indicate if changes were made. 
and $q:(0,+\infty) \rightarrow(0,+\infty), f:[0,+\infty) \times \mathbb{R}^{n+1} \times \mathbb{R}^{n+1} \times \mathbb{R}^{n+1} \times \mathbb{R} \rightarrow \mathbb{R}$ are continuous, and $\tau_{j, i}:[0,+\infty) \rightarrow(0,+\infty)$ are continuous for all $j=0,1,2, i=1,2, \ldots, n$. In what follows we shall always assume that $\lim _{t \rightarrow+\infty}\left(t-\tau_{j, i}(t)\right)=+\infty, j=0,1,2, i=1,2, \ldots, n$. We define the positive real number $\tau$ as

$$
\tau=-\min _{0 \leq j \leq 2,1 \leq i \leq n} \min _{t \geq 0}\left(t-\tau_{j, i}(t)\right)
$$

and seek the solutions of (1.1) which satisfy the boundary conditions

$$
\left\{\begin{array}{l}
u(0)=A, \quad u^{\prime}(0)=B, \quad u^{\prime \prime}(t)-a u^{\prime \prime \prime}(t)=\theta(t), \quad-\tau \leq t \leq 0 \\
u^{\prime \prime \prime}(+\infty)=C
\end{array}\right.
$$

where $\theta \in \mathcal{C}[-\tau, 0], A, B \in \mathbb{R}, a, C \geq 0$, and $u^{\prime \prime \prime}(+\infty)=\lim _{t \rightarrow+\infty} u^{\prime \prime \prime}(t)$. For this, following as in several above works, and inspired by the contributions in [25-30], we shall employ the method of upper and lower solutions.

The plan of this paper is as follows: In Section 2, we state some definitions and lemmas which are needed to prove the main results. In Section 3, we show that in the presence of a pair of upper and lower solutions the problem (1.1)-(1.2) has at least one solution. Also in this section, we establish that in the presence of two pairs of upper and lower solutions the problem (1.1)-(1.2) has at least three solutions. Finally, in Section 4, we illustrate two examples which show the importance of our results.

\section{Preliminaries}

In this section we introduce some necessary definitions, lemmas, and preliminary results that will be used in main results which give the existence of solutions of the problem (1.1)(1.2). First, we construct the Green's function for the linear boundary value problem

$$
\begin{cases}u^{(4)}(t)+e(t)=0, & 0<t<+\infty \\ u(0)=A, \quad u^{\prime}(0)=B, \quad u^{\prime \prime}(t)-a u^{\prime \prime \prime}(t)=\theta(t), & -\tau \leq t \leq 0 \\ u^{\prime \prime \prime}(+\infty)=C & \end{cases}
$$

Lemma 2.1 Let $e \in L^{1}[0,+\infty)$. Then the solution $u \in \mathcal{C}^{3}[-\tau,+\infty) \cap \mathcal{C}^{4}(0,+\infty)$ of the problem (2.1) can be expressed as

$$
u(t)= \begin{cases}\phi(t), & -\tau \leq t \leq 0 \\ A+B t+(a C+\theta(0)) \frac{t^{2}}{2}+C \frac{t^{3}}{3 !}+\int_{0}^{\infty} G(t, s) e(s) d s, & 0 \leq t<+\infty\end{cases}
$$

where

$$
G(t, s)= \begin{cases}\frac{a}{2} t^{2}+\frac{s t^{2}}{2}-\frac{s^{2} t}{2}+\frac{s^{3}}{3 !}, & 0 \leq s \leq t<+\infty \\ \frac{a}{2} t^{2}+\frac{t^{3}}{3 !}, & 0 \leq t \leq s<+\infty\end{cases}
$$

and

$$
\begin{aligned}
\phi(t)= & A+B t+\left(\theta(0)+a C+a \int_{0}^{\infty} e(s) d s\right)\left(-a t-a^{2}+a^{2} e^{\frac{t}{a}}\right) \\
& +\int_{t}^{0}\left(s-a-t+a e^{\frac{t-s}{a}}\right) \theta(s) d s .
\end{aligned}
$$


Proof Since $e \in L^{1}[0,+\infty)$, we can integrate (2.1) from $t$ to $+\infty$, and use $u^{\prime \prime \prime}(+\infty)=C$, to get

$$
u^{\prime \prime \prime}(t)=C+\int_{t}^{\infty} e(s) d s, \quad t \geq 0
$$

Integrating the above equation on $[0, t]$, and applying Fubini's theorem and using $u^{\prime \prime}(0)-$ $a u^{\prime \prime \prime}(0)=\theta(0)$, we obtain

$$
u^{\prime \prime}(t)=a C+a \int_{0}^{\infty} e(s) d s+\theta(0)+C t+\int_{0}^{t} s e(s) d s+\int_{t}^{\infty} t e(s) d s
$$

Again integrating (2.4) twice on $[0, t]$, and applying Fubini's theorem and using $u(0)=A$ and $u^{\prime}(0)=B$, we find

$$
\begin{aligned}
u(t)= & A+B t+(a C+\theta(0)) \frac{t^{2}}{2}+C \frac{t^{3}}{3 !}+\int_{0}^{t}\left(\frac{a}{2} t^{2}+\frac{s t^{2}}{2}-\frac{s^{2} t}{2}+\frac{s^{3}}{3 !}\right) e(s) d s \\
& +\int_{t}^{\infty}\left(\frac{a}{2} t^{2}+\frac{t^{3}}{3 !}\right) e(s) d s,
\end{aligned}
$$

for $t \in[0,+\infty)$. Now we consider the following third order linear differential equation:

$$
u^{\prime \prime}(t)-a u^{\prime \prime \prime}(t)=\theta(t), \quad t \in[-\tau, 0] .
$$

If the above equation is rearranged, we have

$$
u^{\prime \prime \prime}(t)-\frac{1}{a} u^{\prime \prime}(t)=-\frac{1}{a} \theta(t), \quad t \in[-\tau, 0]
$$

and solving this linear equation on $[t, 0]$, we find

$$
u^{\prime \prime}(t)=u^{\prime \prime}(0) e^{\frac{t}{a}}+\frac{1}{a} \int_{t}^{0} e^{\frac{t-s}{a}} \theta(s) d s .
$$

Next, integrating (2.5) twice on $[t, 0]$, and applying Fubini's theorem and using the following boundary conditions:

$$
u(0)=A, \quad u^{\prime}(0)=B \quad \text { and } \quad u^{\prime \prime}(0)=\theta(0)+a u^{\prime \prime \prime}(0)=\theta(0)+a C+a \int_{0}^{\infty} e(s) d s,
$$

we obtain

$$
\begin{aligned}
u(t)= & A+B t+\left(\theta(0)+a C+a \int_{0}^{\infty} e(s) d s\right)\left(-a t-a^{2}+a^{2} e^{\frac{t}{a}}\right) \\
& +\int_{t}^{0}\left(s-a-t+a e^{\frac{t-s}{a}}\right) \theta(s) d s
\end{aligned}
$$

for $t \in[-\tau, 0]$. This completes the proof of the lemma. 
Remark 2.2 $G(t, s)$ defined in (2.3) is the Green's function of the BVP

$$
\left\{\begin{array}{l}
-u^{(4)}(t)=0, \quad 0<t<+\infty \\
u(0)=u^{\prime}(0)=0, \quad u^{\prime \prime}(0)=a u^{\prime \prime \prime}(0), \quad u^{\prime \prime \prime}(+\infty)=0
\end{array}\right.
$$

Lemma 2.3 The Green's function $G(t, s)$ has the following properties:

(1) $G(t, s)$ is twice continuously differentiable on $[0,+\infty) \times[0,+\infty)$ and

$$
\left.\frac{\partial^{3} G(t, s)}{\partial t^{3}}\right|_{t=s^{+}}-\left.\frac{\partial^{3} G(t, s)}{\partial t^{3}}\right|_{t=s^{-}}=-1 ;
$$

(2) $\frac{\partial^{i} G(t, s)}{\partial t^{i}} \geq 0, \forall(t, s) \in[0,+\infty) \times[0,+\infty)$, for $i=0,1,2,3$;

(3) $\sup _{t \in[0,+\infty)} \frac{G(t, s)}{1+t^{3}} \leq\left(\frac{a \sqrt[3]{4}+1}{6}\right), \sup _{t \in[0,+\infty)}\left(\frac{1}{1+t^{2}} \frac{\partial G(t, s)}{\partial t}\right) \leq\left(\frac{a+1}{2}\right)$,

$$
\sup _{t \in[0,+\infty)}\left(\frac{1}{1+t} \frac{\partial^{2} G(t, s)}{\partial t^{2}}\right) \leq(a+1), \sup _{t \in[0,+\infty)} \frac{\partial^{3} G(t, s)}{\partial t^{3}} \leq 1 .
$$

Proof (1) and (2) are obvious. Here we shall prove the first inequality of (3). We note that for all integers $k$ and $l$

$$
\sup _{t \in[0,+\infty)} \frac{t^{k}}{1+t^{l}}= \begin{cases}\frac{l-k}{l}\left(\frac{k}{l-k}\right)^{\frac{k}{l}}, & k<l \\ 1, & k=l \\ +\infty, & k>l\end{cases}
$$

For $s \leq t$, we have

$$
\begin{aligned}
\sup _{t \in[0,+\infty)} \frac{G(t, s)}{1+t^{3}} & =\sup _{t \in[0,+\infty)}\left(\frac{\frac{a}{2} t^{2}+\frac{s t^{2}}{2}-\frac{s^{2} t}{2}+\frac{s^{3}}{6}}{1+t^{3}}\right) \leq \sup _{t \in[0,+\infty)}\left(\frac{\frac{a t^{2}}{2}}{1+t^{3}}+\frac{\frac{t^{3}}{6}}{1+t^{3}}\right) \\
& \leq \frac{a}{2} \sup _{t \in[0,+\infty)} \frac{t^{2}}{1+t^{3}}+\frac{1}{6} \sup _{t \in[0,+\infty)} \frac{t^{3}}{1+t^{3}} \leq \frac{a \sqrt[3]{4}+1}{6}
\end{aligned}
$$

and for $s \geq t$

$$
\begin{aligned}
\sup _{t \in[0,+\infty)} \frac{G(t, s)}{1+t^{3}} & =\sup _{t \in[0,+\infty)}\left(\frac{\frac{a}{2} t^{2}+\frac{t^{3}}{6}}{1+t^{3}}\right) \leq \sup _{t \in[0,+\infty)}\left(\frac{\frac{a t^{2}}{2}}{1+t^{3}}+\frac{\frac{t^{3}}{6}}{1+t^{3}}\right) \\
& \leq \frac{a}{2} \sup _{t \in[0,+\infty)} \frac{t^{2}}{1+t^{3}}+\frac{1}{6} \sup _{t \in[0,+\infty)} \frac{t^{3}}{1+t^{3}} \leq \frac{a \sqrt[3]{4}+1}{6} .
\end{aligned}
$$

The other parts can be proved similarly.

We consider the space $X$ defined by

$$
\begin{aligned}
X= & \left\{u \in C^{3}[-\tau,+\infty): \sup _{t \in[0,+\infty)} \frac{|u(t)|}{1+t^{3}}<+\infty, \sup _{t \in[0,+\infty)} \frac{\left|u^{\prime}(t)\right|}{1+t^{2}}<+\infty,\right. \\
& \left.\sup _{t \in[0,+\infty)} \frac{\left|u^{\prime \prime}(t)\right|}{1+t}<+\infty, \lim _{t \rightarrow+\infty} u^{\prime \prime \prime}(t) \text { exists }\right\}
\end{aligned}
$$

with the norm

$$
\|u\|=\max \left\{\|u\|_{0},\|u\|_{1},\|u\|_{2},\|u\|_{\infty}^{0},\|u\|_{\infty}^{1},\|u\|_{\infty}^{2},\|u\|_{\infty}^{3}\right\},
$$


where

$$
\begin{array}{ll}
\|u\|_{0}=\max _{t \in[-\tau, 0]}|u(t)|, \quad\|u\|_{\infty}^{0}=\sup _{t \in[0,+\infty)} \frac{|u(t)|}{1+t^{3}}, \\
\|u\|_{1}=\max _{t \in[-\tau, 0]}\left|u^{\prime}(t)\right|, \quad\|u\|_{\infty}^{1}=\sup _{t \in[0,+\infty)} \frac{\left|u^{\prime}(t)\right|}{1+t^{2}}, \\
\|u\|_{2}=\max _{t \in[-\tau, 0]}\left|u^{\prime \prime}(t)\right|, \quad\|u\|_{\infty}^{2}=\sup _{t \in[0,+\infty)} \frac{\left|u^{\prime \prime}(t)\right|}{1+t}, \quad\|u\|_{\infty}^{3}=\sup _{t \in[-\tau,+\infty)}\left|u^{\prime \prime \prime}(t)\right| .
\end{array}
$$

It is clear that $(X,\|\cdot\|)$ is a Banach space. Next we define the mapping $T: X \rightarrow$ $\mathcal{C}^{3}[-\tau,+\infty) \cap \mathcal{C}^{4}(0,+\infty)$ by

$$
T u(t)= \begin{cases}\psi(t), & -\tau \leq t \leq 0 \\ l(t)+\int_{0}^{\infty} G(t, s) q(s) f\left(s,[u(s)],\left[u^{\prime}(s)\right],\left[u^{\prime \prime}(s)\right], u^{\prime \prime \prime}(s)\right) d s, & 0 \leq t<+\infty\end{cases}
$$

where

$$
\begin{aligned}
\psi(t)= & A+B t+\left(\theta(0)+a C+a \int_{0}^{\infty} q(s) f\left(s,[u(s)],\left[u^{\prime}(s)\right],\left[u^{\prime \prime}(s)\right], u^{\prime \prime \prime}(s)\right) d s\right) \\
& \times\left(-a t-a^{2}+a^{2} e^{\frac{t}{a}}\right)+\int_{t}^{0}\left(s-a-t+a e^{\frac{t-s}{a}}\right) \theta(s) d s
\end{aligned}
$$

and

$$
l(t)=A+B t+(a C+\theta(0)) \frac{t^{2}}{2}+C \frac{t^{3}}{3 !} .
$$

Lemma 2.4 The mapping $T: X \rightarrow \mathcal{C}^{3}[-\tau,+\infty) \cap \mathcal{C}^{4}(0,+\infty)$ in (2.6) has the following properties:

(1) $T u(0)=A,(T u)^{\prime}(0)=B,(T u)^{\prime \prime}(t)-a(T u)^{\prime \prime \prime}(t)=\theta(t)$ for $t \in[-\tau, 0]$,

(2) $\mathrm{Tu}(t)$ is three-times continuously differentiable on $t \in[-\tau,+\infty)$,

(3) $(T u)^{(4)}(t)=-q(t) f\left(t,[u(t)],\left[u^{\prime}(t)\right],\left[u^{\prime \prime}(t)\right], u^{\prime \prime \prime}(t)\right), t \in(0,+\infty)$,

(4) fixed points of $T$ are solutions of $B V P$ (1.1)-(1.2).

When applying the Schäuder fixed point theorem to prove the existence result, it is necessary to show that the operator $T_{1}$ (defined later) is completely continuous. For this, we need the following modified version of the Arzela-Ascoli lemma (see $[18,20])$.

Lemma 2.5 $M \subset X$ is relatively compact if the following conditions hold:

(1) all functions belonging to $M$ are uniformly bounded,

(2) all functions belonging to $M$ are equi-continuous on any compact sub-interval of $[-\tau,+\infty)$,

(3) all functions from $M$ are equi-convergent at infinity, that is, for any $\epsilon>0$, there exists a $T=T(\epsilon)>0$ such that, for all $t \geq T$ and any $u \in M$,

$$
\begin{aligned}
& \left|\frac{u(t)}{1+t^{3}}-\lim _{t \rightarrow+\infty} \frac{u(t)}{1+t^{3}}\right|<\epsilon, \quad\left|\frac{u^{\prime}(t)}{1+t^{2}}-\lim _{t \rightarrow+\infty} \frac{u^{\prime}(t)}{1+t^{2}}\right|<\epsilon, \\
& \left|\frac{u^{\prime \prime}(t)}{1+t}-\lim _{t \rightarrow+\infty} \frac{u^{\prime \prime}(t)}{1+t}\right|<\epsilon \quad \text { and } \quad\left|u^{\prime \prime \prime}(t)-\lim _{t \rightarrow+\infty} u^{\prime \prime \prime}(t)\right|<\epsilon .
\end{aligned}
$$


Definition 2.6 A function $\alpha \in X \cap \mathcal{C}^{4}(0,+\infty)$ is called a lower solution of (1.1)-(1.2) provided

$$
\begin{aligned}
& \alpha^{(4)}(t)+q(t) f\left(t,[\alpha(t)],\left[\alpha^{\prime}(t)\right],\left[\alpha^{\prime \prime}(t)\right], \alpha^{\prime \prime \prime}(t)\right) \geq 0, \quad 0<t<+\infty ; \\
& \alpha(0) \leq A, \quad \alpha^{\prime}(0)=B, \quad \alpha^{\prime \prime}(t)-a \alpha^{\prime \prime \prime}(t) \leq \theta(t), \quad-\tau \leq t \leq 0 ; \\
& \alpha^{\prime \prime \prime}(+\infty) \leq C .
\end{aligned}
$$

Similarly, a function $\beta \in X \cap \mathcal{C}^{4}(0,+\infty)$ is called an upper solution of (1.1)-(1.2) provided

$$
\begin{aligned}
& \beta^{(4)}(t)+q(t) f\left(t,[\beta(t)],\left[\beta^{\prime}(t)\right],\left[\beta^{\prime \prime}(t)\right], \beta^{\prime \prime \prime}(t)\right) \leq 0, \quad 0<t<+\infty ; \\
& \beta(0) \geq A, \quad \beta^{\prime}(0)=B, \quad \beta^{\prime \prime}(t)-a \beta^{\prime \prime \prime}(t) \geq \theta(t), \quad-\tau \leq t \leq 0 ; \\
& \beta^{\prime \prime \prime}(+\infty) \geq C .
\end{aligned}
$$

Also, we say $\alpha(\beta)$ is a strict lower solution (strict upper solution) for problem (1.1)-(1.2) if all the above inequalities are strict.

\section{Remark 2.7 If}

$$
\alpha^{\prime \prime}(t) \leq \beta^{\prime \prime}(t), \quad \text { for every } t \in[-\tau,+\infty)
$$

then on integrating (2.12) and using the boundary restrictions in Definition 2.6, we find that $\alpha^{\prime}(t) \leq \beta^{\prime}(t), \alpha(t) \leq \beta(t)$ for all $t \in[0,+\infty)$ and $\beta^{\prime}(t) \leq \alpha^{\prime}(t), \alpha(t) \leq \beta(t)$ for all $t \in$ $[-\tau, 0)$.

Definition 2.8 Let $\alpha, \beta$ be lower and upper solutions for the problem (1.1)-(1.2) satisfying

$$
\alpha^{\prime \prime}(t) \leq \beta^{\prime \prime}(t), \quad \text { for all } t \in[-\tau,+\infty) \text {. }
$$

A continuous function $f$ is said to satisfy Nagumo's condition with respect to the pair of functions $\alpha, \beta$ if there exist positive functions $\varphi$ and $h \in \mathcal{C}[0,+\infty)$ such that

$$
\left|f\left(t, x_{0}, \ldots, x_{n}, y_{0}, \ldots, y_{n}, z_{0}, \ldots, z_{n}, w\right)\right| \leq \varphi(t) h(|w|)
$$

for all $t \in[0,+\infty)$, and $\left(x_{0}, \ldots, x_{n}\right) \in[[\alpha(t)],[\beta(t)]], y_{i}\left(t-\tau_{1, i}(t)\right) \in\left[\alpha^{\prime}\left(t-\tau_{1, i}(t)\right), \beta^{\prime}(t-\right.$ $\left.\left.\tau_{1, i}(t)\right)\right]$ if $t-\tau_{1, i}(t)>0, y_{i}\left(t-\tau_{1, i}(t)\right) \in\left[\beta^{\prime}\left(t-\tau_{1, i}(t)\right), \alpha^{\prime}\left(t-\tau_{1, i}(t)\right)\right]$ if $t-\tau_{1, i}(t) \leq 0,0 \leq i \leq n$, $\tau_{1,0}=0,\left(z_{0}, \ldots, z_{n}\right) \in\left[\left[\alpha^{\prime \prime}(t)\right],\left[\beta^{\prime \prime}(t)\right]\right], w \in \mathbb{R}$, and

$$
\int_{0}^{\infty} q(s) \varphi(s) d s<+\infty, \quad \int_{0}^{\infty} \frac{s}{h(s)} d s=+\infty
$$

\section{Main results}

In this section we state and prove our existence results. We begin with the following lemma.

Lemma 3.1 Suppose the following conditions hold. 
$\left(\mathrm{H}_{1}\right)$ BVP (1.1)-(1.2) has a pair of lower and upper solutions $\alpha, \beta$ satisfying

$$
\alpha^{\prime \prime}(t) \leq \beta^{\prime \prime}(t), \quad \text { for } t \in[-\tau,+\infty)
$$

and $f$ satisfies Nagumo's condition with respect to the pair of functions $\alpha, \beta$.

$\left(\mathrm{H}_{2}\right)$ There exists a constant $\gamma>1$ such that

$$
\sup _{0 \leq t<+\infty}(1+t)^{\gamma} q(t) \varphi(t)<+\infty
$$

where $\varphi$ is the function in Nagumo's condition off.

Then there exists a constant $R>0$ (depending on $\alpha, \beta, h$, and $C$ ) such that every solution $u$ of (1.1)-(1.2) with

$$
\begin{array}{ll}
\alpha(t) \leq u(t) \leq \beta(t), & \alpha^{\prime}(t) \leq u^{\prime}(t) \leq \beta^{\prime}(t), \\
\alpha^{\prime \prime}(t) \leq u^{\prime \prime}(t) \leq \beta^{\prime \prime}(t) & \text { for all } t \in[0,+\infty)
\end{array}
$$

and

$$
\begin{array}{ll}
\alpha(t) \leq u(t) \leq \beta(t), & \beta^{\prime}(t) \leq u^{\prime}(t) \leq \alpha^{\prime}(t), \\
\alpha^{\prime \prime}(t) \leq u^{\prime \prime}(t) \leq \beta^{\prime \prime}(t) & \text { for all } t \in[-\tau, 0)
\end{array}
$$

satisfies $\|u\|_{\infty}^{3}<R$.

Proof We can choose $R>\eta$ such that

$$
\eta \geq \max \left\{\sup _{t \in[0,+\infty)}\left|\beta^{\prime \prime \prime}(t)\right|, \sup _{t \in[0,+\infty)}\left|\alpha^{\prime \prime \prime}(t)\right|, \frac{\left\|\beta^{\prime \prime}-\theta\right\|_{0}}{a}, \frac{\left\|\alpha^{\prime \prime}-\theta\right\|_{0}}{a}, C\right\}
$$

and

$$
\int_{\eta}^{R} \frac{s}{h(s)} d s>M\left(\sup _{t \in[0,+\infty)} \frac{\beta^{\prime \prime}(t)}{(1+t)^{\gamma}}-\inf _{t \in[0,+\infty)} \frac{\alpha^{\prime \prime}(t)}{(1+t)^{\gamma}}+\frac{\gamma N}{\gamma-1}\right),
$$

where $C$ is the nonhomogeneous boundary value, and

$$
M=\sup _{t \in[0,+\infty)}(1+t)^{\gamma} q(t) \varphi(t), \quad N=\max \left\{\|\beta\|_{\infty}^{2},\|\alpha\|_{\infty}^{2}\right\}
$$

Let $u$ be a solution of the differential equation (1.1) satisfying (3.1) and (3.2). If $\left|u^{\prime \prime \prime}(t)\right|<R$, for all $t \in[0,+\infty)$, there is nothing to prove. If this is not true, there exists a $t_{0} \in[0,+\infty)$ such that $\left|u^{\prime \prime \prime}\left(t_{0}\right)\right| \geq R$. Since $\lim _{t \rightarrow+\infty} u^{\prime \prime \prime}(t)=C<R$, there exists a $T>0$ such that

$$
\left|u^{\prime \prime \prime}(t)\right|<R \text { for all } t \geq T \text {. }
$$

Let $t_{1}=\inf \left\{t \leq T:\left|u^{\prime \prime \prime}(s)\right|<R\right.$ for all $\left.s \in[t,+\infty)\right\}$. Then $\left|u^{\prime \prime \prime}\left(t_{1}\right)\right|=R$ and $\left|u^{\prime \prime \prime}(t)\right|<R$ for all $t>t_{1}$ and there exists a $t_{2}$ such that $\left|u^{\prime \prime \prime}(t)\right| \geq R$ for $t \in\left[t_{2}, t_{1}\right]$. So we have two cases 
to consider $u^{\prime \prime \prime}\left(t_{1}\right)=R$ and $u^{\prime \prime \prime}(t) \geq R$ for $t \in\left[t_{2}, t_{1}\right]$, or $u^{\prime \prime \prime}\left(t_{1}\right)=-R$ and $u^{\prime \prime \prime}(t) \leq-R$ for $t \in\left[t_{2}, t_{1}\right]$. We assume that $u^{\prime \prime \prime}\left(t_{1}\right)=R$ and $u^{\prime \prime \prime}(t) \geq R$ for $t \in\left[t_{2}, t_{1}\right]$, then we have

$$
\begin{aligned}
\int_{\eta}^{R} \frac{s}{h(s)} d s & \leq \int_{C}^{R} \frac{s}{h(s)} d s \\
& =-\int_{t_{1}}^{\infty} \frac{u^{\prime \prime \prime}(s) u^{(4)}(s)}{h\left(u^{\prime \prime \prime}(s)\right)} d s \\
& =-\int_{t_{1}}^{\infty} \frac{-q(s) f\left(s,[u(s)],\left[u^{\prime}(s)\right],\left[u^{\prime \prime}(s)\right], u^{\prime \prime \prime}(s)\right) u^{\prime \prime \prime}(s)}{h\left(u^{\prime \prime \prime}(s)\right)} d s \\
& \leq \int_{t_{1}}^{\infty} q(s) \varphi(s) u^{\prime \prime \prime}(s) d s \\
& \leq M \int_{t_{1}}^{\infty} \frac{u^{\prime \prime \prime}(s)}{(1+s)^{\gamma}} d s \\
& =M\left(\int_{t_{1}}^{\infty}\left(\frac{u^{\prime \prime}(s)}{(1+s)^{\gamma}}\right)^{\prime} d s+\int_{t_{1}}^{\infty} \frac{u^{\prime \prime}(s)}{1+s} \cdot \frac{\gamma}{(1+s)^{\gamma}} d s\right) \\
& \leq M\left(\sup _{t \in[0,+\infty)} \frac{\beta^{\prime \prime}(t)}{(1+t)^{\gamma}}-\inf _{t \in[0,+\infty)} \frac{\alpha^{\prime \prime}(t)}{(1+t)^{\gamma}}+\frac{\gamma N}{\gamma-1}\right) \\
& <\int_{\eta}^{R} \frac{s}{h(s)} d s,
\end{aligned}
$$

which is a contradiction. In the case $u^{\prime \prime \prime}\left(t_{1}\right)=-R$ and $u^{\prime \prime \prime}(t) \leq-R$ for $t \in\left[t_{2}, t_{1}\right]$, we obtain a similar contradiction. Thus, $\left|u^{\prime \prime \prime}(t)\right|<R$ for all $t \in[0,+\infty)$. From the boundary condition (1.2) we also have

$$
-R<-\eta \leq \frac{\alpha^{\prime \prime}(t)-\theta(t)}{a} \leq u^{\prime \prime \prime}(t)=\frac{u^{\prime \prime}(t)-\theta(t)}{a} \leq \frac{\beta^{\prime \prime}(t)-\theta(t)}{a} \leq \eta<R
$$

for all $t \in[-\tau, 0]$. Therefore, $\left|u^{\prime \prime \prime}(t)\right|<R$ for $t \in[-\tau, 0)$. To sum up, we have $\|u\|_{\infty}^{3}<R$.

Theorem 3.2 Suppose conditions $\left(\mathrm{H}_{1}\right)$ and $\left(\mathrm{H}_{2}\right)$ hold. Suppose further that

$\left(\mathrm{H}_{3}\right)$ For any fixed $t \in[0,+\infty), y_{i}, z_{i}, w \in \mathbb{R}, i=0, \ldots, n$, when

$$
\begin{aligned}
& \alpha\left(t-\tau_{0, i}(t)\right) \leq x_{i} \leq \beta\left(t-\tau_{0, i}(t)\right), \quad i=0,1, \ldots, n \\
& \quad f\left(t, x_{0}, x_{1}, \ldots, \alpha\left(t-\tau_{0, i}(t)\right), \ldots, x_{n}, y_{0}, \ldots, y_{n}, z_{0}, \ldots, z_{n}, w\right) \\
& \quad \leq f\left(t, x_{0}, x_{1}, \ldots, x_{i}, \ldots, x_{n}, y_{0}, \ldots, y_{n}, z_{0}, \ldots, z_{n}, w\right) \\
& \quad \leq f\left(t, x_{0}, x_{1}, \ldots, \beta\left(t-\tau_{0, i}(t)\right), \ldots, x_{n}, y_{0}, \ldots, y_{n}, z_{0}, \ldots, z_{n}, w\right) .
\end{aligned}
$$

$\left(\mathrm{H}_{4}\right)$ For any fixed $t \in[0,+\infty), x_{i}, z_{i}, w \in \mathbb{R}, i=0, \ldots, n$ when

$$
\alpha^{\prime}\left(t-\tau_{1, i}(t)\right) \leq y_{i} \leq \beta^{\prime}\left(t-\tau_{1, i}(t)\right), \quad t-\tau_{1, i}(t)>0,
$$

or when

$$
\beta^{\prime}\left(t-\tau_{1, i}(t)\right) \leq y_{i} \leq \alpha^{\prime}\left(t-\tau_{1, i}(t)\right), \quad t-\tau_{1, i}(t) \leq 0, i=0,1, \ldots, n,
$$




$$
\begin{aligned}
& f\left(t, x_{0}, \ldots, x_{n}, y_{0}, \ldots, \alpha^{\prime}\left(t-\tau_{1, i}(t)\right), \ldots, y_{n}, z_{0}, \ldots, z_{n}, w\right) \\
& \quad \leq f\left(t, x_{0}, \ldots, x_{n}, y_{0}, \ldots, y_{i}, \ldots, y_{n}, z_{0}, \ldots, z_{n}, w\right) \\
& \quad \leq f\left(t, x_{0}, \ldots, x_{n}, y_{0}, \ldots, \beta^{\prime}\left(t-\tau_{1, i}(t)\right), \ldots, y_{n}, z_{0}, \ldots, z_{n}, w\right) .
\end{aligned}
$$

$\left(\mathrm{H}_{5}\right)$ For any fixed $t \in[0,+\infty), x_{i}, y_{i}, w \in \mathbb{R}, i=0, \ldots, n$ when

$$
\begin{aligned}
& \alpha^{\prime \prime}\left(t-\tau_{2, i}(t)\right) \leq z_{i} \leq \beta^{\prime \prime}\left(t-\tau_{2, i}(t)\right), \quad i=0,1, \ldots, n \\
& f\left(t, x_{0}, \ldots, x_{n}, y_{0}, \ldots, y_{n}, z_{0}, \ldots, \alpha^{\prime \prime}\left(t-\tau_{2, i}(t)\right), \ldots, z_{n}, w\right) \\
& \quad \leq f\left(t, x_{0}, \ldots, x_{n}, y_{0}, \ldots, y_{n}, z_{0}, \ldots, z_{i}, \ldots, z_{n}, w\right) \\
& \quad \leq f\left(t, x_{0}, \ldots, x_{n}, y_{0}, \ldots, y_{n}, z_{0}, \ldots, \beta^{\prime \prime}\left(t-\tau_{2, i}(t)\right), \ldots, z_{n}, w\right)
\end{aligned}
$$

where $\tau_{0,0}=\tau_{1,0}=\tau_{2,0}=0$.

$\left(\mathrm{H}_{6}\right)$

$$
\int_{0}^{\infty} \max \{s, 1\} q(s) d s<+\infty, \quad \int_{0}^{\infty} \max \{s, 1\} q(s) \varphi(s) d s<+\infty
$$

Then BVP (1.1)-(1.2) has at least one solution $u \in X \cap \mathcal{C}^{4}(0,+\infty)$ satisfying (3.1)-(3.2) and $\|u\|_{\infty}^{3}<R$.

Proof Let $R$ be a positive number as in Lemma 3.1 and define the auxiliary functions,

$$
F_{0}, F_{1}, F_{2}, F_{3}:[0,+\infty) \times \mathbb{R}^{n+1} \times \mathbb{R}^{n+1} \times \mathbb{R}^{n+1} \times \mathbb{R} \rightarrow \mathbb{R}
$$

as follows:

$$
\begin{aligned}
& F_{0}\left(t, x_{0}, \ldots, x_{n}, y_{0}, \ldots, y_{n}, z_{0}, \ldots, z_{n}, w\right) \\
& = \begin{cases}f\left(t, \beta, \widetilde{x}_{1}, \ldots, \tilde{x}_{n}, y_{0}, \ldots, y_{n}, z_{0}, \ldots, z_{n}, w\right), & x_{0}>\beta(t) \\
f\left(t, x_{0}, \widetilde{x}_{1}, \ldots, \tilde{x}_{n}, y_{0}, \ldots, y_{n}, z_{0}, \ldots, z_{n}, w\right), & \alpha(t) \leq x_{0} \leq \beta(t), \\
f\left(t, \alpha, \tilde{x}_{1}, \ldots, \widetilde{x}_{n}, y_{0}, \ldots, y_{n}, z_{0}, \ldots, z_{n}, w\right), & x_{0}<\alpha(t)\end{cases} \\
& F_{1}\left(t, x_{0}, \ldots, x_{n}, y_{0}, \ldots, y_{n}, z_{0}, \ldots, z_{n}, w\right) \\
& = \begin{cases}F_{0}\left(t, x_{0}, \ldots, x_{n}, \beta^{\prime}, \tilde{y}_{1}, \ldots, \tilde{y}_{n}, z_{0}, \ldots, z_{n}, w\right), & y_{0}>\beta^{\prime}(t), \\
F_{0}\left(t, x_{0}, \ldots, x_{n}, y_{0}, \tilde{y}_{1}, \ldots, \tilde{y}_{n}, z_{0}, \ldots, z_{n}, w\right), & \alpha^{\prime}(t) \leq y_{0} \leq \beta^{\prime}(t), \\
F_{0}\left(t, x_{0}, \ldots, x_{n}, \alpha^{\prime}, \tilde{y}_{1}, \ldots, \tilde{y}_{n}, z_{0}, \ldots, z_{n}, w\right), & y_{0}<\alpha^{\prime}(t),\end{cases} \\
& F_{2}\left(t, x_{0}, \ldots, x_{n}, y_{0}, \ldots, y_{n}, z_{0}, \ldots, z_{n}, w\right) \\
& = \begin{cases}F_{1}\left(t, x_{0}, \ldots, x_{n}, y_{0}, \ldots, y_{n}, \beta^{\prime \prime}, \widetilde{z}_{1}, \ldots, \widetilde{z}_{n}, w\right)-\frac{z_{0}-\beta^{\prime \prime}}{1+\left|z_{0}-\beta^{\prime \prime}\right|}, & z_{0}>\beta^{\prime \prime}(t), \\
F_{1}\left(t, x_{0}, \ldots, x_{n}, y_{0}, \ldots, y_{n}, z_{0}, \widetilde{z}_{1}, \ldots, \widetilde{z}_{n}, w\right), & \alpha^{\prime \prime}(t) \leq z_{0} \leq \beta^{\prime \prime}(t), \\
F_{1}\left(t, x_{0}, \ldots, x_{n}, y_{0}, \ldots, y_{n}, \alpha^{\prime \prime}, \widetilde{z}_{1}, \ldots, \widetilde{z}_{n}, w\right)+\frac{z_{0}-\alpha^{\prime \prime}}{1+\left|z_{0}-\alpha^{\prime \prime}\right|}, & z_{0}<\alpha^{\prime \prime}(t),\end{cases} \\
& F_{3}\left(t, x_{0}, x_{1}, \ldots, x_{n}, y_{0}, y_{1}, \ldots, y_{n}, z_{0}, \ldots, z_{n}, w\right) \\
& = \begin{cases}F_{2}\left(t, x_{0}, \ldots, x_{n}, y_{0}, \ldots, y_{n}, z_{0}, \ldots, z_{n}, R\right), & w>R \\
F_{2}\left(t, x_{0}, \ldots, x_{n}, y_{0}, \ldots, y_{n}, z_{0}, \ldots, z_{n}, w\right), & -R \leq w \leq R \\
F_{2}\left(t, x_{0}, \ldots, x_{n}, y_{0}, \ldots, y_{n}, z_{0}, \ldots, z_{n},-R\right), & w<R\end{cases}
\end{aligned}
$$


where, for $i=1,2, \ldots, n$,

$$
\tilde{x}_{i}= \begin{cases}\beta, & x_{i}>\beta\left(t-\tau_{0, i}(t)\right) \\ x_{i}, & \alpha\left(t-\tau_{0, i}(t)\right) \leq x_{i} \leq \beta\left(t-\tau_{0, i}(t)\right) ; \\ \alpha, & x_{i}<\alpha\left(t-\tau_{0, i}(t)\right)\end{cases}
$$

if $t-\tau_{1, i}(t)>0$,

$$
\tilde{y}_{i}= \begin{cases}\beta^{\prime}, & y_{i}>\beta^{\prime}\left(t-\tau_{1, i}(t)\right) \\ y_{i}, & \alpha^{\prime}\left(t-\tau_{1, i}(t)\right) \leq y_{i} \leq \beta^{\prime}\left(t-\tau_{1, i}(t)\right) \\ \alpha^{\prime}, & y_{i}<\alpha^{\prime}\left(t-\tau_{1, i}(t)\right)\end{cases}
$$

if $t-\tau_{1, i}(t) \leq 0$,

$$
\tilde{y}_{i}= \begin{cases}\alpha^{\prime}, & y_{i}>\alpha^{\prime}\left(t-\tau_{1, i}(t)\right) \\ y_{i}, & \beta^{\prime}\left(t-\tau_{1, i}(t)\right) \leq y_{i} \leq \alpha^{\prime}\left(t-\tau_{1, i}(t)\right) \\ \beta^{\prime}, & y_{i}<\beta^{\prime}\left(t-\tau_{1, i}(t)\right)\end{cases}
$$

and

$$
\widetilde{z}_{i}= \begin{cases}\beta^{\prime \prime}, & z_{i}>\beta^{\prime \prime}\left(t-\tau_{2, i}(t)\right) \\ z_{i}, & \alpha^{\prime \prime}\left(t-\tau_{2, i}(t)\right) \leq z_{i} \leq \beta^{\prime \prime}\left(t-\tau_{2, i}(t)\right) \\ \alpha^{\prime \prime}, & z_{i}<\alpha^{\prime \prime}\left(t-\tau_{2, i}(t)\right)\end{cases}
$$

We consider the modified boundary value problem

$$
u^{(4)}(t)+q(t) F_{3}\left(t,[u(t)],\left[u^{\prime}(t)\right],\left[u^{\prime \prime}(t)\right], u^{\prime \prime \prime}(t)\right)=0, \quad 0<t<+\infty,
$$

with the boundary conditions (1.2). We will show that the problem (3.3)-(1.2) has at least one solution $u$ in $X$. Now for $u \in X$, we define two operators $\widetilde{T}_{1}, T_{1}$ by

$$
\widetilde{T}_{1} u(t)=\int_{0}^{\infty} G(t, s) q(s) F_{3}\left(s,[u(s)],\left[u^{\prime}(s)\right],\left[u^{\prime \prime}(s)\right], u^{\prime \prime \prime}(s)\right) d s
$$

and

$$
T_{1} u(t)= \begin{cases}\psi_{1}(t), & -\tau \leq t \leq 0 \\ l(t)+\widetilde{T}_{1} u(t), & 0 \leq t<+\infty\end{cases}
$$

where $l(t)$ is as in (2.7) and

$$
\begin{aligned}
\psi_{1}(t)= & A+B t+\left(\theta(0)+a C+a \int_{0}^{\infty} q(s) F_{3}\left(s,[u(s)],\left[u^{\prime}(s)\right],\left[u^{\prime \prime}(s)\right], u^{\prime \prime \prime}(s)\right) d s\right) \\
& \times\left(-a t-a^{2}+a^{2} e^{\frac{t}{a}}\right)+\int_{t}^{0}\left(s-a-t+a e^{\frac{t-s}{a}}\right) \theta(s) d s .
\end{aligned}
$$

We want to show that the operator $T_{1}$ is completely continuous. We split the proof in the following parts: 
(1) $T_{1}: X \rightarrow X$ is well defined. Obviously, for any $u \in X$ by direct calculation, we have

$$
\begin{aligned}
& \left(T_{1} u\right)^{\prime \prime}(t)-a\left(T_{1} u\right)^{\prime \prime \prime}(t)=\theta(t) \quad \text { for } t \in[-\tau, 0] \quad \text { and } \\
& \left(T_{1} u\right)^{\prime}(0)=B, \quad\left(T_{1} u\right)(0)=A
\end{aligned}
$$

and for $t \in(0,+\infty)$,

$$
\begin{aligned}
& \left(T_{1} u\right)^{\prime}(t)=l^{\prime}(t)+\int_{0}^{\infty} \frac{\partial G(t, s)}{\partial t} q(s) F_{3}\left(s,[u(s)],\left[u^{\prime}(s)\right],\left[u^{\prime \prime}(s)\right], u^{\prime \prime \prime}(s)\right) d s, \\
& \left(T_{1} u\right)^{\prime \prime}(t)=l^{\prime \prime}(t)+\int_{0}^{\infty} \frac{\partial^{2} G(t, s)}{\partial t^{2}} q(s) F_{3}\left(s,[u(s)],\left[u^{\prime}(s)\right],\left[u^{\prime \prime}(s)\right], u^{\prime \prime \prime}(s)\right) d s, \\
& \left(T_{1} u\right)^{\prime \prime \prime}(t)=C+\int_{t}^{\infty} q(s) F_{3}\left(s,[u(s)],\left[u^{\prime}(s)\right],\left[u^{\prime \prime}(s)\right], u^{\prime \prime \prime}(s)\right) d s,
\end{aligned}
$$

which show that $T_{1} u(t) \in C^{3}[-\tau,+\infty)$. Further, we have

$$
\begin{gathered}
\left|\int_{0}^{\infty} q(s) F_{3}\left(s,[u(s)],\left[u^{\prime}(s)\right],\left[u^{\prime \prime}(s)\right], u^{\prime \prime \prime}(s)\right) d s\right| \\
\leq \int_{0}^{\infty} \max \{s, 1\} q(s)(H \varphi(s)+1) d s<+\infty
\end{gathered}
$$

where $H=\max _{0 \leq s \leq \sup _{t \in[0,+\infty)}\left|u^{\prime \prime \prime}(t)\right|} h(s)$. Now from (3.5) it follows that

$$
\int_{1}^{\infty} s q(s)(H \varphi(s)+1) d s \leq \int_{0}^{\infty} \max \{s, 1\} q(s)(H \varphi(s)+1) d s<+\infty
$$

which implies

$$
\lim _{t \rightarrow+\infty} t q(t)(H \varphi(t)+1)=0
$$

Next since

$$
\int_{t}^{\infty} q(s)(H \varphi(s)+1) d s \leq \int_{t}^{\infty} s q(s)(H \varphi(s)+1) d s<+\infty, \quad t \geq 1
$$

we also have

$$
\lim _{t \rightarrow+\infty} \int_{t}^{\infty} q(s)(H \varphi(s)+1) d s=0
$$

By Lebesgue's dominated convergent theorem, L'Hopital's rule, and (3.6), (3.7), we obtain

$$
\begin{aligned}
& \left|\lim _{t \rightarrow+\infty} \frac{\left(\widetilde{T}_{1} u\right)(t)}{1+t^{3}}\right| \\
& \quad \leq \lim _{t \rightarrow+\infty} \int_{0}^{\infty} \frac{|G(t, s)|}{1+t^{3}} q(s)\left|F_{3}\left(s,[u(s)],\left[u^{\prime}(s)\right],\left[u^{\prime \prime}(s)\right], u^{\prime \prime \prime}(s)\right)\right| d s \\
& \quad \leq \lim _{t \rightarrow+\infty}\left(\int_{0}^{\infty} \frac{|G(t, s)|}{1+t^{3}} q(s)(H \varphi(s)+1) d s\right)
\end{aligned}
$$




$$
\begin{aligned}
& =\lim _{t \rightarrow+\infty}\left[\int_{0}^{t} \frac{\left(\frac{a}{2} t^{2}+\frac{s t^{2}}{2}-\frac{s^{2} t}{2}+\frac{s^{3}}{6}\right)}{1+t^{3}} q(s)(H \varphi(s)+1) d s\right. \\
& \left.+\int_{t}^{\infty} \frac{\left(\frac{a}{2} t^{2}+\frac{t^{3}}{6}\right)}{1+t^{3}} q(s)(H \varphi(s)+1) d s\right] \\
& =\lim _{t \rightarrow+\infty}\left[\int_{0}^{t} \frac{\left(a t+s t-\frac{s^{2}}{2}\right)}{3 t^{2}} q(s)(H \varphi(s)+1) d s+\frac{\left(\frac{a}{2} t^{2}+\frac{t^{3}}{6}\right)}{3 t^{2}} q(t)(H \varphi(t)+1)\right] \\
& +\lim _{t \rightarrow+\infty}\left[\int_{t}^{\infty} \frac{\left(a t+\frac{t^{2}}{2}\right)}{3 t^{2}} q(s)(H \varphi(s)+1) d s-\frac{\left(\frac{a}{2} t^{2}+\frac{t^{3}}{6}\right)}{3 t^{2}} q(t)(H \varphi(t)+1)\right] \\
& =\lim _{t \rightarrow+\infty} \int_{0}^{t} \frac{\left(a t+s t-\frac{s^{2}}{2}\right)}{3 t^{2}} q(s)(H \varphi(s)+1) d s+\lim _{t \rightarrow+\infty} \frac{\left(\frac{a}{2} t+\frac{t^{2}}{6}\right)}{3 t^{2}} t q(t)(H \varphi(t)+1) \\
& +\lim _{t \rightarrow+\infty} \int_{t}^{\infty} \frac{\left(a t+\frac{t^{2}}{2}\right)}{3 t^{2}} q(s)(H \varphi(s)+1) d s-\lim _{t \rightarrow+\infty} \frac{\left(\frac{a}{2} t+\frac{t^{2}}{6}\right)}{3 t^{2}} t q(t)(H \varphi(t)+1) \\
& =\lim _{t \rightarrow+\infty} \int_{0}^{t}\left[\frac{(a+s)}{6 t} q(s)(H \varphi(s)+1) d s+\frac{\left(a t+\frac{t^{2}}{2}\right)}{6 t} q(t)(H \varphi(t)+1)\right] \\
& +\lim _{t \rightarrow+\infty}\left[\int_{t}^{\infty} \frac{a+t}{6 t} q(s)(H \varphi(s)+1) d s-\frac{\left(a t+\frac{t^{2}}{2}\right)}{6 t} q(t)(H \varphi(t)+1)\right] \\
& =\lim _{t \rightarrow+\infty} \int_{0}^{t} \frac{(a+s)}{6 t} q(s)(H \varphi(s)+1) d s+\lim _{t \rightarrow+\infty} \frac{\left(a+\frac{t}{2}\right)}{6 t} t q(t)(H \varphi(t)+1) \\
& +\lim _{t \rightarrow+\infty} \int_{t}^{\infty} \frac{a+t}{6 t} q(s)(H \varphi(s)+1) d s-\lim _{t \rightarrow+\infty} \frac{\left(a+\frac{t}{2}\right)}{6 t} t q(t)(H \varphi(t)+1) \\
& =\lim _{t \rightarrow+\infty} \int_{t}^{\infty} q(s)(H \varphi(s)+1) d s-\lim _{t \rightarrow+\infty} \frac{(a+t)}{6} q(t)(H \varphi(t)+1) \\
& =\lim _{t \rightarrow+\infty} \int_{t}^{\infty} q(s)(H \varphi(s)+1) d s-\lim _{t \rightarrow+\infty} \frac{(a+t)}{6 t} t q(t)(H \varphi(t)+1) \\
& =\frac{1}{6} \lim _{t \rightarrow+\infty} \int_{t}^{\infty} q(s)(H \varphi(s)+1) d s=0,
\end{aligned}
$$

that is, $\lim _{t \rightarrow+\infty} \frac{\left(\widetilde{T}_{1} u\right)(t)}{1+t^{3}}=0$, and

$$
\begin{aligned}
\lim _{t \rightarrow+\infty} \frac{\left(T_{1} u\right)(t)}{1+t^{3}} & =\lim _{t \rightarrow+\infty} \frac{l(t)}{1+t^{3}}+\lim _{t \rightarrow+\infty} \frac{\left(\widetilde{T}_{1} u\right)(t)}{1+t^{3}} \\
& =\lim _{t \rightarrow+\infty} \frac{A+B t+(a C+\theta(0)) \frac{t^{2}}{2}+C \frac{t^{3}}{3 !}}{1+t^{3}}=\frac{C}{6}
\end{aligned}
$$

which implies that $\sup _{t \in[0,+\infty)} \frac{\left|\left(T_{1} u\right)(t)\right|}{1+t^{3}}<+\infty$. Similarly, we have

$$
\begin{aligned}
& \left|\lim _{t \rightarrow+\infty} \frac{\left(\widetilde{T}_{1} u\right)^{\prime}(t)}{1+t^{2}}\right| \\
& \quad \leq \lim _{t \rightarrow+\infty} \frac{1}{1+t^{2}} \int_{0}^{\infty} \frac{\partial G(t, s)}{\partial t} q(s)\left|F_{3}\left(s,[u(s)],\left[u^{\prime}(s)\right],\left[u^{\prime \prime}(s)\right], u^{\prime \prime \prime}(s)\right)\right| d s \\
& \quad \leq \frac{1}{2} \lim _{t \rightarrow+\infty} \int_{t}^{\infty} q(s)(H \varphi(s)+1) d s=0,
\end{aligned}
$$


that is, $\lim _{t \rightarrow+\infty} \frac{\left(\widetilde{T}_{1} u\right)^{\prime}(t)}{1+t^{2}}=0$ and $\lim _{t \rightarrow+\infty} \frac{\left(\widetilde{T}_{1} u\right)^{\prime}(t)}{1+t^{2}}=\lim _{t \rightarrow+\infty} \frac{l^{\prime}(t)}{1+t^{2}}+\lim _{t \rightarrow+\infty} \frac{\left(T_{1} u\right)^{\prime}(t)}{1+t^{2}}=\frac{C}{2}$, which implies that $\sup _{t \in[0,+\infty)} \frac{\left|\left(T_{1} u\right)^{\prime}(t)\right|}{1+t^{2}}<+\infty$,

$$
\begin{aligned}
\left|\lim _{t \rightarrow+\infty} \frac{\left(\widetilde{T}_{1} u\right)^{\prime \prime}(t)}{1+t}\right| & \leq \lim _{t \rightarrow+\infty} \frac{1}{1+t} \int_{0}^{\infty} \frac{\partial^{2} G(t, s)}{\partial t^{2}} q(s)\left|F_{3}\left(s,[u(s)],\left[u^{\prime}(s)\right],\left[u^{\prime \prime}(s)\right], u^{\prime \prime \prime}(s)\right)\right| d s \\
& \leq \lim _{t \rightarrow+\infty} \int_{t}^{\infty} q(s)(H \varphi(s)+1) d s=0,
\end{aligned}
$$

that is, $\lim _{t \rightarrow+\infty} \frac{\left(\widetilde{T}_{1} u\right)^{\prime \prime}(t)}{1+t}=0$ and $\lim _{t \rightarrow+\infty} \frac{\left(T_{1} u\right)^{\prime \prime}(t)}{1+t}=\lim _{t \rightarrow+\infty} \frac{l^{\prime \prime}(t)}{1+t}+\lim _{t \rightarrow+\infty} \frac{\left(\widetilde{T}_{1} u\right)^{\prime \prime}(t)}{1+t}=C$, which implies that $\sup _{t \in[0,+\infty)} \frac{\left|\left(T_{1} u\right)^{\prime \prime}(t)\right|}{1+t}<+\infty$, and by $(3.5)$

$$
\begin{aligned}
& \left|\lim _{t \rightarrow+\infty} \int_{t}^{\infty} q(s) F_{3}\left(s,[u(s)],\left[u^{\prime}(s)\right],\left[u^{\prime \prime}(s)\right], u^{\prime \prime \prime}(s)\right) d s\right| \\
& \quad \leq \lim _{t \rightarrow+\infty} \int_{t}^{\infty} q(s)(H \varphi(s)+1) d s=0,
\end{aligned}
$$

then

$$
\begin{aligned}
\lim _{t \rightarrow+\infty}\left(T_{1} u\right)^{\prime \prime \prime}(t) & =\lim _{t \rightarrow+\infty}\left(C+\int_{t}^{\infty} q(s) F_{3}\left(s,[u(s)],\left[u^{\prime}(s)\right],\left[u^{\prime \prime}(s)\right], u^{\prime \prime \prime}(s)\right) d s\right) \\
& =C<+\infty .
\end{aligned}
$$

Therefore $T_{1} u \in X$.

(2) $T_{1}: X \rightarrow X$ is continuous. For any convergent sequence $u_{m} \rightarrow u$ in $X$, we have

$$
\begin{aligned}
& u_{m}(t) \rightarrow u(t), \quad u_{m}^{\prime}(t) \rightarrow u^{\prime}(t), \quad u_{m}^{\prime \prime}(t) \rightarrow u^{\prime \prime}(t), \\
& u_{m}^{\prime \prime \prime}(t) \rightarrow u^{\prime \prime \prime}(t), \quad m \rightarrow+\infty, t \in[-\tau,+\infty) .
\end{aligned}
$$

Thus the continuity of $F_{3}$ implies that

$$
\begin{aligned}
& \left|F_{3}\left(s,\left[u_{m}(s)\right],\left[u_{m}^{\prime}(s)\right],\left[u_{m}^{\prime \prime}(s)\right], u_{m}^{\prime \prime \prime}(s)\right)-F_{3}\left(s,[u(s)],\left[u^{\prime}(s)\right],\left[u^{\prime \prime}(s)\right], u^{\prime \prime \prime}(s)\right)\right| \rightarrow 0, \\
& \quad m \rightarrow+\infty
\end{aligned}
$$

Since $u_{m}^{\prime \prime \prime}(t) \rightarrow u^{\prime \prime \prime}(t), \sigma=\sup \left\{s_{1}: s_{1}=\sup _{t \in[0,+\infty)}\left|u_{m}^{\prime \prime \prime}(t)\right|, m \in N\right\}<+\infty$.

Let $H_{1}=\max _{0 \leq s \leq \max \left\{\sup _{t \in[0,+\infty)}\left|u^{\prime \prime \prime}(t)\right|, \sigma\right\}} h(s)$. Then we have

$$
\begin{gathered}
\int_{0}^{\infty} q(s) \mid\left(F_{3}\left(s,\left[u_{m}(s)\right],\left[u_{m}^{\prime}(s)\right],\left[u_{m}^{\prime \prime}(s)\right], u_{m}^{\prime \prime \prime}(s)\right)\right. \\
\left.\quad-F_{3}\left(s,[u(s)],\left[u^{\prime}(s)\right],\left[u^{\prime \prime}(s)\right], u^{\prime \prime \prime}(s)\right)\right) \mid d s \\
\leq 2 \int_{0}^{\infty} q(s)\left(H_{1} \phi(s)+1\right) d s<+\infty .
\end{gathered}
$$

Thus, we find

$$
\begin{aligned}
& \left\|T_{1} u_{m}-T_{1} u\right\|_{0} \\
& \quad=\max _{t \in[-\tau, 0]}\left|\left(T_{1} u_{m}\right)(t)-\left(T_{1} u\right)(t)\right|
\end{aligned}
$$




$$
\begin{aligned}
= & \max _{t \in[-\tau, 0]} \mid \int_{0}^{\infty}\left(-a^{3}-a^{2} t+a^{3} e^{\frac{t}{a}}\right) q(s)\left(F_{3}\left(s,\left[u_{m}(s)\right],\left[u_{m}^{\prime}(s)\right],\left[u_{m}^{\prime \prime}(s)\right], u_{m}^{\prime \prime \prime}(s)\right)\right. \\
& \left.-F_{3}\left(s,[u(s)],\left[u^{\prime}(s)\right],\left[u^{\prime \prime}(s)\right], u^{\prime \prime \prime}(s)\right)\right) d s \mid \\
\leq & \left|-a^{3}+a^{2} \tau+a^{3} e^{\frac{-\tau}{a}}\right| \int_{0}^{\infty} q(s) \mid F_{3}\left(s,\left[u_{m}(s)\right],\left[u_{m}^{\prime}(s)\right],\left[u_{m}^{\prime \prime}(s)\right], u_{m}^{\prime \prime \prime}(s)\right) \\
& -F_{3}\left(s,[u(s)],\left[u^{\prime}(s)\right],\left[u^{\prime \prime}(s)\right], u^{\prime \prime \prime}(s)\right) \mid d s,
\end{aligned}
$$

that is,

$$
\left\|T_{1} u_{m}-T_{1} u\right\|_{0} \rightarrow 0
$$

as $m \rightarrow+\infty$.

$$
\begin{aligned}
\left\|T_{1} u_{m}-T_{1} u\right\|_{\infty}^{0}= & \sup _{t \in[0,+\infty)}\left|\frac{\left(\widetilde{T}_{1} u_{m}\right)(t)}{1+t^{3}}-\frac{\left(\widetilde{T}_{1} u\right)(t)}{1+t^{3}}\right| \\
= & \sup _{t \in[0,+\infty)} \mid \int_{0}^{\infty} \frac{G(t, s)}{1+t^{3}} q(s)\left(F_{3}\left(s,\left[u_{m}(s)\right],\left[u_{m}^{\prime}(s)\right],\left[u_{m}^{\prime \prime}(s)\right], u_{m}^{\prime \prime \prime}(s)\right)\right. \\
& \left.-F_{3}\left(s,[u(s)],\left[u^{\prime}(s)\right],\left[u^{\prime \prime}(s)\right], u^{\prime \prime \prime}(s)\right)\right) d s \mid \\
\leq & \int_{0}^{\infty}\left(\frac{a \sqrt[3]{4}+1}{6}\right) q(s) \mid F_{3}\left(s,\left[u_{m}(s)\right],\left[u_{m}^{\prime}(s)\right],\left[u_{m}^{\prime \prime}(s)\right], u_{m}^{\prime \prime \prime}(s)\right) \\
& -F_{3}\left(s,[u(s)],\left[u^{\prime}(s)\right],\left[u^{\prime \prime}(s)\right], u^{\prime \prime \prime}(s)\right) \mid d s,
\end{aligned}
$$

that is,

$$
\left\|T_{1} u_{m}-T_{1} u\right\|_{\infty}^{0} \rightarrow 0
$$

as $m \rightarrow+\infty$.

$$
\begin{aligned}
\left\|T_{1} u_{m}-T_{1} u\right\|_{1}= & \max _{t \in[-\tau, 0]}\left|\left(T_{1} u_{m}\right)^{\prime}(t)-\left(T_{1} u\right)^{\prime}(t)\right| \\
= & \max _{t \in[-\tau, 0]} \mid \int_{0}^{\infty}\left(-a^{2}+a^{2} e^{\frac{t}{a}}\right) q(s)\left(F_{3}\left(s,\left[u_{m}(s)\right],\left[u_{m}^{\prime}(s)\right],\left[u_{m}^{\prime \prime}(s)\right], u_{m}^{\prime \prime \prime}(s)\right)\right. \\
& \left.-F_{3}\left(s,[u(s)],\left[u^{\prime}(s)\right],\left[u^{\prime \prime}(s)\right], u^{\prime \prime \prime}(s)\right)\right) d s \mid \\
\leq & \left|-a^{2}+a^{2} e^{\frac{-\tau}{a}}\right| \int_{0}^{\infty} q(s) \mid F_{3}\left(s,\left[u_{m}(s)\right],\left[u_{m}^{\prime}(s)\right],\left[u_{m}^{\prime \prime}(s)\right], u_{m}^{\prime \prime \prime}(s)\right) \\
& -F_{3}\left(s,[u(s)],\left[u^{\prime}(s)\right],\left[u^{\prime \prime}(s)\right], u^{\prime \prime \prime}(s)\right) \mid d s,
\end{aligned}
$$

that is,

$$
\left\|T_{1} u_{m}-T_{1} u\right\|_{1} \rightarrow 0
$$




$$
\begin{aligned}
\left\|T_{1} u_{m}-T_{1} u\right\|_{\infty}^{1} \\
=\sup _{t \in[0,+\infty)}\left|\frac{\left(\widetilde{T}_{1} u_{m}\right)^{\prime}(t)}{1+t^{2}}-\frac{\left(\widetilde{T}_{1} u\right)^{\prime}(t)}{1+t^{2}}\right| \\
=\sup _{t \in[0,+\infty)} \mid \frac{1}{1+t^{2}} \int_{0}^{\infty} \frac{\partial G(t, s)}{\partial t} q(s)\left(F_{3}\left(s,\left[u_{m}(s)\right],\left[u_{m}^{\prime}(s)\right],\left[u_{m}^{\prime \prime}(s)\right], u_{m}^{\prime \prime \prime}(s)\right)\right. \\
\left.\quad-F_{3}\left(s,[u(s)],\left[u^{\prime}(s)\right],\left[u^{\prime \prime}(s)\right], u^{\prime \prime \prime}(s)\right)\right) d s \mid \\
\leq \int_{0}^{\infty}\left(\frac{a+1}{2}\right) q(s) \mid F_{3}\left(s,\left[u_{m}(s)\right],\left[u_{m}^{\prime}(s)\right],\left[u_{m}^{\prime \prime}(s)\right], u_{m}^{\prime \prime \prime}(s)\right) \\
\quad-F_{3}\left(s,[u(s)],\left[u^{\prime}(s)\right],\left[u^{\prime \prime}(s)\right], u^{\prime \prime \prime}(s)\right) \mid d s,
\end{aligned}
$$

that is,

$$
\left\|T_{1} u_{m}-T_{1} u\right\|_{\infty}^{1} \rightarrow 0
$$

as $m \rightarrow+\infty$.

$$
\begin{aligned}
\left\|T_{1} u_{m}-T_{1} u\right\|_{2}= & \max _{t \in[-\tau, 0]}\left|\left(T_{1} u_{m}\right)^{\prime \prime}(t)-\left(T_{1} u\right)^{\prime \prime}(t)\right| \\
= & \max _{t \in[-\tau, 0]} \mid \int_{0}^{\infty} a e^{\frac{t}{a}} q(s)\left(F_{3}\left(s,\left[u_{m}(s)\right],\left[u_{m}^{\prime}(s)\right],\left[u_{m}^{\prime \prime}(s)\right], u_{m}^{\prime \prime \prime}(s)\right)\right. \\
& \left.-F_{3}\left(s,[u(s)],\left[u^{\prime}(s)\right],\left[u^{\prime \prime}(s)\right], u^{\prime \prime \prime}(s)\right)\right) d s \mid \\
\leq & a \int_{0}^{\infty} q(s) \mid F_{3}\left(s,\left[u_{m}(s)\right],\left[u_{m}^{\prime}(s)\right],\left[u_{m}^{\prime \prime}(s)\right], u_{m}^{\prime \prime \prime}(s)\right) \\
& -F_{3}\left(s,[u(s)],\left[u^{\prime}(s)\right],\left[u^{\prime \prime}(s)\right], u^{\prime \prime \prime}(s)\right) \mid d s,
\end{aligned}
$$

that is,

$$
\left\|T_{1} u_{m}-T_{1} u\right\|_{2} \rightarrow 0
$$

as $m \rightarrow+\infty$.

$$
\begin{aligned}
\| & T_{1} u_{m}-T_{1} u \|_{\infty}^{2} \\
= & \sup _{t \in[0,+\infty)}\left|\frac{\left(\widetilde{T}_{1} u_{m}\right)^{\prime \prime}(t)}{1+t}-\frac{\left(\widetilde{T}_{1} u\right)^{\prime \prime}(t)}{1+t}\right| \\
= & \sup _{t \in[0,+\infty)} \mid \frac{1}{1+t} \int_{0}^{\infty} \frac{\partial^{2} G(t, s)}{\partial t^{2}} q(s)\left(F_{3}\left(s,\left[u_{m}(s)\right],\left[u_{m}^{\prime}(s)\right],\left[u_{m}^{\prime \prime}(s)\right], u_{m}^{\prime \prime \prime}(s)\right)\right. \\
& \left.-F_{3}\left(s,[u(s)],\left[u^{\prime}(s)\right],\left[u^{\prime \prime}(s)\right], u^{\prime \prime \prime}(s)\right)\right) d s \mid \\
\leq & \int_{0}^{\infty}(a+1) q(s) \mid F_{3}\left(s,\left[u_{m}(s)\right],\left[u_{m}^{\prime}(s)\right],\left[u_{m}^{\prime \prime}(s)\right], u_{m}^{\prime \prime \prime}(s)\right) \\
& -F_{3}\left(s,[u(s)],\left[u^{\prime}(s)\right],\left[u^{\prime \prime}(s)\right], u^{\prime \prime \prime}(s)\right) \mid d s,
\end{aligned}
$$


that is,

$$
\left\|T_{1} u_{m}-T_{1} u\right\|_{\infty}^{2} \rightarrow 0
$$

as $m \rightarrow+\infty$.

To show $\left\|T_{1} u_{m}-T_{1} u\right\|_{\infty}^{3} \rightarrow 0$, as $m \rightarrow+\infty$, we need the following:

$$
\begin{aligned}
& \sup _{t \in[0,+\infty)}\left|\left(T_{1} u_{m}\right)^{\prime \prime \prime}(t)-\left(T_{1} u\right)^{\prime \prime \prime}(t)\right| \\
& =\sup _{t \in[0,+\infty)}\left|\left(\widetilde{T}_{1} u_{m}\right)^{\prime \prime \prime}(t)-\left(\widetilde{T}_{1} u\right)^{\prime \prime \prime}(t)\right| \\
& =\sup _{t \in[0,+\infty)} \mid \int_{t}^{\infty} q(s)\left(F_{3}\left(s,\left[u_{m}(s)\right],\left[u_{m}^{\prime}(s)\right],\left[u_{m}^{\prime \prime}(s)\right], u_{m}^{\prime \prime \prime}(s)\right)\right. \\
& \left.\quad-F_{3}\left(s,[u(s)],\left[u^{\prime}(s)\right],\left[u^{\prime \prime}(s)\right], u^{\prime \prime \prime}(s)\right)\right) d s \mid \\
& \leq \int_{0}^{\infty} q(s) \mid F_{3}\left(s,\left[u_{m}(s)\right],\left[u_{m}^{\prime}(s)\right],\left[u_{m}^{\prime \prime}(s)\right], u_{m}^{\prime \prime \prime}(s)\right) \\
& \quad-F_{3}\left(s,[u(s)],\left[u^{\prime}(s)\right],\left[u^{\prime \prime}(s)\right], u^{\prime \prime \prime}(s)\right) \mid d s
\end{aligned}
$$

and

$$
\begin{aligned}
& \sup _{t \in[-\tau, 0)}\left|\left(T_{1} u_{m}\right)^{\prime \prime \prime}(t)-\left(T_{1} u\right)^{\prime \prime \prime}(t)\right| \\
& =\sup _{t \in[-\tau, 0)} \frac{1}{a}\left|\left(T_{1} u_{m}\right)^{\prime \prime}(t)-\left(T_{1} u\right)^{\prime \prime}(t)\right| \\
& \leq \int_{0}^{\infty} q(s) \mid F_{3}\left(s,\left[u_{m}(s)\right],\left[u_{m}^{\prime}(s)\right],\left[u_{m}^{\prime \prime}(s)\right], u_{m}^{\prime \prime \prime}(s)\right) \\
& \quad-F_{3}\left(s,[u(s)],\left[u^{\prime}(s)\right],\left[u^{\prime \prime}(s)\right], u^{\prime \prime \prime}(s)\right) \mid d s .
\end{aligned}
$$

Hence, it follows that

$$
\begin{aligned}
& \left\|T_{1} u_{m}-T_{1} u\right\|_{\infty}^{3} \\
& =\sup _{t \in[-\tau,+\infty)}\left|\left(T_{1} u_{m}\right)^{\prime \prime \prime}(t)-\left(T_{1} u\right)^{\prime \prime \prime}(t)\right| \\
& \left.\leq \sup _{t \in[0,+\infty)} \mid\left(T_{1} u_{m}\right)^{\prime \prime \prime}(t)-T_{1} u\right)^{\prime \prime \prime}(t)\left|+\sup _{t \in[-\tau, 0)}\right|\left(T_{1} u_{m}\right)^{\prime \prime \prime}(t)-\left(T_{1} u\right)^{\prime \prime \prime}(t) \mid \\
& \leq 2 \int_{0}^{\infty} q(s) \mid F_{3}\left(s,\left[u_{m}(s)\right],\left[u_{m}^{\prime}(s)\right],\left[u_{m}^{\prime \prime}(s)\right], u_{m}^{\prime \prime \prime}(s)\right) \\
& \quad-F_{3}\left(s,[u(s)],\left[u^{\prime}(s)\right],\left[u^{\prime \prime}(s)\right], u^{\prime \prime \prime}(s)\right) \mid d s,
\end{aligned}
$$

that is,

$$
\left\|T_{1} u_{m}-T_{1} u\right\|_{\infty}^{3} \rightarrow 0
$$


Combining (3.8)-(3.14), we find $\left\|\left(T_{1} u_{m}\right)-\left(T_{1} u\right)\right\| \rightarrow 0$, as $m \rightarrow+\infty$; so $T_{1}: X \rightarrow X$ is continuous.

(3) $T_{1}: X \rightarrow X$ is compact. The operator $T_{1}$ is compact if $T_{1}$ maps bounded subsets of $X$ into relatively compact sets. Let $K$ be any bounded subset of $X$, then $r_{3}=$ $\sup _{0 \leq s \leq\left\{\sup _{t \in[0,+\infty)}\left|u^{\prime \prime \prime}(t)\right|, u \in K\right\}} h(s)<+\infty$. For any $u \in K$, we have the following:

$$
\begin{aligned}
&\left\|T_{1} u\right\|_{0} \leq|A|+\tau|B|+\left(\left|a^{2} C+a \theta(0)\right|+\tau\|\theta\|_{0}\right)\left(-a+\tau+a e^{\frac{-\tau}{a}}\right) \\
&+\left(-a^{3}+a^{2} \tau+a^{3} e^{\frac{-\tau}{a}}\right) \int_{0}^{\infty} q(s)\left(r_{3} \varphi(s)+1\right) d s, \\
&\left\|T_{1} u\right\|_{\infty}^{0} \leq|A|+|B|+|a C+\theta(0)|+C+\left(\frac{a \sqrt[3]{4}+1}{6}\right) \int_{0}^{\infty} q(s)\left(r_{3} \varphi(s)+1\right) d s, \\
&\left\|\left(T_{1} u\right)\right\|_{1} \leq|B|+\left(\left|a^{2} C+a \theta(0)\right|+\tau\|\theta\|_{0}\right)\left(1-e^{\frac{-\tau}{a}}\right) \\
&+\left(a^{2}-a^{2} e^{\frac{-\tau}{a}}\right) \int_{0}^{\infty} q(s)\left(r_{3} \varphi(s)+1\right) d s, \\
&\left\|\left(T_{1} u\right)\right\|_{\infty}^{1} \leq|B|+|a C+\theta(0)|+C+\left(\frac{a+2}{2}\right) \int_{0}^{\infty} q(s)\left(r_{3} \varphi(s)+1\right) d s, q(s)\left(r_{3} \varphi(s)+1\right) d s, \\
&\left\|\left(T_{1} u\right)\right\|_{2} \leq|a C+\theta(0)|+\|\theta\|_{0}+a \int_{0}^{\infty} \\
&\left\|\left(T_{1} u\right)\right\|_{\infty}^{2} \leq|a C+\theta(0)|+C+(a+1) \int_{0}^{\infty} q(s)\left(r_{3} \varphi(s)+1\right) d s, \\
&\left\|\left(T_{1} u\right)\right\|_{\infty}^{3} \leq \frac{1}{a}|a C+\theta(0)|+\frac{2}{a}\|\theta\|_{0}+C+2 \int_{0}^{\infty} q(s)\left(r_{3} \varphi(s)+1\right) d s,
\end{aligned}
$$

which implies that

$$
\left\|T_{1} u\right\| \leq|A|+\xi|B|+v|a C+\theta(0)|+C+\gamma\|\theta\|_{0}+\chi \int_{0}^{\infty} q(s)\left(r_{3} \varphi(s)+1\right) d s,
$$

where

$$
\begin{aligned}
& \chi=\max \left\{\left(-a^{3}+a^{2} \tau+a^{3} e^{\frac{-\tau}{a}}\right),\left(\frac{a \sqrt[3]{4}+1}{6}\right),\left(a^{2}-a^{2} e^{\frac{-\tau}{a}}\right),(a+1), 2\right\}, \\
& v=\max \left\{\left(-a^{2}+a \tau+a^{2} e^{\frac{-\tau}{a}}\right),\left(a-a e^{\frac{-\tau}{a}}\right), \frac{1}{a}, 1\right\}, \quad \xi=\max \{\tau, 1\}, \\
& \gamma=\max \left\{\left(-a \tau+\tau^{2}+a \tau e^{\frac{-\tau}{a}}\right),\left(\tau-\tau e^{\frac{-\tau}{a}}\right), 1, \frac{2}{a}\right\} .
\end{aligned}
$$

Therefore, $T_{1} K$ is uniformly bounded. We also know that $\psi_{1}(t)$ and $\psi_{1}^{\prime}(t)$ are continuous on $[-\tau, 0]$. Thus in view of $[-\tau, 0]$ compact, $\psi_{1}(t)$ and $\psi_{1}^{\prime}(t)$ are also uniformly continuous. Thus it follows that for $t_{1}, t_{2} \in[-\tau, 0]$,

$$
\begin{aligned}
& \left|T_{1} u\left(t_{1}\right)-T_{1} u\left(t_{2}\right)\right|=\left|\psi_{1}\left(t_{1}\right)-\psi_{1}\left(t_{2}\right)\right| \rightarrow 0 \text { as } t_{1} \rightarrow t_{2}, \\
& \left|\left(T_{1} u\right)^{\prime}\left(t_{1}\right)-\left(T_{1} u\right)^{\prime}\left(t_{2}\right)\right|=\left|\psi_{1}^{\prime}\left(t_{1}\right)-\psi_{1}^{\prime}\left(t_{2}\right)\right| \rightarrow 0 \text { as } t_{1} \rightarrow t_{2},
\end{aligned}
$$


further since

$$
\begin{aligned}
\left(T_{1} u\right)^{\prime \prime}(t)= & \psi_{1}^{\prime \prime}(t) \\
= & \left(\theta(0)+a C+a \int_{0}^{\infty} q(s) F_{3}\left(s,[u(s)],\left[u^{\prime}(s)\right],\left[u^{\prime \prime}(s)\right], u^{\prime \prime \prime}(s)\right) d s\right) e^{\frac{t}{a}} \\
& +\frac{1}{a} \int_{t}^{0} e^{\frac{t-s}{a}} \theta(s) d s
\end{aligned}
$$

is continuous on $[-\tau, 0]$, we find

$$
\left|\left(T_{1} u\right)^{\prime \prime}\left(t_{1}\right)-\left(T_{1} u\right)^{\prime \prime}\left(t_{2}\right)\right|=\left|\psi_{1}^{\prime \prime}\left(t_{1}\right)-\psi_{1}^{\prime \prime}\left(t_{2}\right)\right| \rightarrow 0 \quad \text { as } t_{1} \rightarrow t_{2} .
$$

Next, for $t_{1}, t_{2} \in[0, \varepsilon]$ with $\varepsilon>0$ a constant, we have

$$
\begin{aligned}
& \left|\frac{\left(T_{1} u\right)\left(t_{1}\right)}{1+t_{1}^{3}}-\frac{\left(T_{1} u\right)\left(t_{2}\right)}{1+t_{2}^{3}}\right| \\
& =\mid \frac{l\left(t_{1}\right)}{1+t_{1}^{3}}-\frac{l\left(t_{2}\right)}{1+t_{2}^{3}}+\int_{0}^{\infty}\left(\frac{G\left(t_{1}, s\right)}{1+t_{1}^{3}}-\frac{G\left(t_{2}, s\right)}{1+t_{2}^{3}}\right) q(s) \\
& \times F_{3}\left(s,[u(s)],\left[u^{\prime}(s)\right],\left[u^{\prime \prime}(s)\right], u^{\prime \prime \prime}(s)\right) d s \\
& \leq\left|\frac{l\left(t_{1}\right)}{1+t_{1}^{3}}-\frac{l\left(t_{2}\right)}{1+t_{2}^{3}}\right|+\int_{0}^{\infty}\left|\frac{G\left(t_{1}, s\right)}{1+t_{1}^{3}}-\frac{G\left(t_{2}, s\right)}{1+t_{2}^{3}}\right| q(s)\left(r_{3} \varphi(s)+1\right) d s \\
& \rightarrow 0 \quad \text { as } t_{1} \rightarrow t_{2} \text {, } \\
& \left|\frac{\left(T_{1} u\right)^{\prime}\left(t_{1}\right)}{1+t_{1}^{2}}-\frac{\left(T_{1} u\right)^{\prime}\left(t_{2}\right)}{1+t_{2}^{2}}\right| \\
& =\mid \frac{l^{\prime}\left(t_{1}\right)}{1+t_{1}^{2}}-\frac{l^{\prime}\left(t_{2}\right)}{1+t_{2}^{2}}+\int_{0}^{\infty}\left(\frac{\frac{\partial G\left(t_{1}, s\right)}{\partial t}}{1+t_{1}^{2}}-\frac{\frac{\partial G\left(t_{2}, s\right)}{\partial t}}{1+t_{2}^{2}}\right) q(s) \\
& \times F_{3}\left(s,[u(s)],\left[u^{\prime}(s)\right],\left[u^{\prime \prime}(s)\right], u^{\prime \prime \prime}(s)\right) d s \\
& \leq\left|\frac{l^{\prime}\left(t_{1}\right)}{1+t_{1}^{2}}-\frac{l^{\prime}\left(t_{2}\right)}{1+t_{2}^{2}}\right|+\int_{0}^{\infty}\left|\frac{\frac{\partial G\left(t_{1}, s\right)}{\partial t}}{1+t_{1}^{2}}-\frac{\frac{\partial G\left(t_{2}, s\right)}{\partial t}}{1+t_{2}^{2}}\right| q(s)\left(r_{3} \varphi(s)+1\right) d s \\
& \rightarrow 0 \quad \text { as } t_{1} \rightarrow t_{2} \text {, } \\
& \left|\frac{\left(T_{1} u\right)^{\prime \prime}\left(t_{1}\right)}{1+t_{1}}-\frac{\left(T_{1} u\right)^{\prime \prime}\left(t_{2}\right)}{1+t_{2}}\right| \\
& =\mid \frac{l^{\prime \prime}\left(t_{1}\right)}{1+t_{1}}-\frac{l^{\prime \prime}\left(t_{2}\right)}{1+t_{2}}+\int_{0}^{\infty}\left(\frac{\frac{\partial^{2} G\left(t_{1}, s\right)}{\partial t^{2}}}{1+t_{1}^{2}}-\frac{\frac{\partial^{2} G\left(t_{2}, s\right)}{\partial} t^{2}}{1+t_{2}^{2}}\right) q(s) \\
& \times F_{3}\left(s,[u(s)],\left[u^{\prime}(s)\right],\left[u^{\prime \prime}(s)\right], u^{\prime \prime \prime}(s)\right) d s \\
& \leq\left|\frac{l^{\prime \prime}\left(t_{1}\right)}{1+t_{1}}-\frac{l^{\prime \prime}\left(t_{2}\right)}{1+t_{2}}\right|+\int_{0}^{\infty}\left|\frac{\frac{\partial^{2} G\left(t_{1}, s\right)}{\partial t^{2}}}{1+t_{1}}-\frac{\frac{\partial^{2} G\left(t_{2}, s\right)}{\partial t^{2}}}{1+t_{2}}\right| q(s)\left(r_{3} \varphi(s)+1\right) d s \\
& \rightarrow 0 \quad \text { as } t_{1} \rightarrow t_{2} \text {, }
\end{aligned}
$$


and

$$
\begin{aligned}
& \left|\left(T_{1} u\right)^{\prime \prime \prime}\left(t_{1}\right)-\left(T_{1} u\right)^{\prime \prime \prime}\left(t_{2}\right)\right| \\
& =\mid \int_{t_{1}}^{\infty} q(s) F_{3}\left(s,[u(s)],\left[u^{\prime}(s)\right],\left[u^{\prime \prime}(s)\right], u^{\prime \prime \prime}(s)\right) d s \\
& \quad-\int_{t_{2}}^{\infty} q(s) F_{3}\left(s,[u(s)],\left[u^{\prime}(s)\right],\left[u^{\prime \prime}(s)\right], u^{\prime \prime \prime}(s)\right) d s \mid \\
& \leq \int_{t_{1}}^{t_{2}} q(s)\left(r_{3} \varphi(s)+1\right) d s \\
& \rightarrow 0 \quad \text { as } t_{1} \rightarrow t_{2} .
\end{aligned}
$$

Thus, $T_{1} K$ is equi-continuous. Finally, we will show that $T_{1} K$ is equi-convergent at infinity. In fact, when $t>0$ we have

$$
\begin{aligned}
& \left|\frac{\left(T_{1} u\right)(t)}{1+t^{3}}-\lim _{t \rightarrow+\infty} \frac{\left(T_{1} u\right)(t)}{1+t^{3}}\right|=\left|\frac{\left(T_{1} u\right)(t)}{1+t^{3}}-\frac{C}{6}\right| \rightarrow 0, \quad \text { as } t \rightarrow+\infty, \\
& \left|\frac{\left(T_{1} u\right)^{\prime}(t)}{1+t^{2}}-\lim _{t \rightarrow+\infty} \frac{\left(T_{1} u\right)^{\prime}(t)}{1+t^{2}}\right|=\left|\frac{\left(T_{1} u\right)^{\prime}(t)}{1+t^{2}}-\frac{C}{2}\right| \rightarrow 0, \quad \text { as } t \rightarrow+\infty, \\
& \left|\frac{\left(T_{1} u\right)^{\prime \prime}(t)}{1+t}-\lim _{t \rightarrow+\infty} \frac{\left(T_{1} u\right)^{\prime \prime}(t)}{1+t}\right|=\left|\frac{\left(T_{1} u\right)^{\prime \prime}(t)}{1+t}-C\right| \rightarrow 0, \quad \text { as } t \rightarrow+\infty,
\end{aligned}
$$

and

$$
\begin{aligned}
& \left|\left(T_{1} u\right)^{\prime \prime \prime}(t)-\lim _{t \rightarrow+\infty}\left(T_{1} u\right)^{\prime \prime \prime}(t)\right|=\left|\left(T_{1} u\right)^{\prime \prime \prime}(t)-C\right| \leq\left|\int_{t}^{\infty} q(s)\left(r_{3} \varphi(s)+1\right) d s\right| \rightarrow 0 \\
& \quad \text { as } t \rightarrow+\infty
\end{aligned}
$$

Hence all conditions of Lemma 2.5 are fulfilled, so $T_{1} K$ is relatively compact. Therefore, $T_{1}: X \rightarrow X$ is completely continuous.

(4) $T_{1}: X \rightarrow X$ has at least one fixed point. Let $\Omega=\{u \in X,\|u\| \leq N\}$ where

$$
N=|A|+\xi|B|+v|a C+\theta(0)|+C+\gamma\|\theta\|_{0}+\chi \int_{0}^{\infty} q(s)(H \varphi(s)+1) d s
$$

For any $u \in \Omega$, it is easy to see that $\left\|T_{1} u\right\| \leq \Omega$, and thus $T_{1} \Omega \subset \Omega$. The Schäuder fixed point theorem now guarantees that the operator $T_{1}$ has at least one fixed point in $\Omega$, which is a solution of BVP (3.3)-(1.2). Now we shall show that this solution $u$ satisfies the inequalities (3.1) and (3.2) which in view of the definitions of $F_{3}, F_{2}, F_{1}$, and $F_{0}$ will imply that $u$ is in fact a solution of (1.1)-(1.2). For this, we only prove that $u^{\prime \prime}(t) \leq \beta^{\prime \prime}(t), t \in[-\tau,+\infty)$. A similar argument can be used to prove $\alpha^{\prime \prime}(t) \leq u^{\prime \prime}(t), t \in[-\tau,+\infty)$. If not true, we set $\omega(t)=u^{\prime \prime}(t)-\beta^{\prime \prime}(t)$, then there exists $t^{*} \in[-\tau,+\infty)$ such that $\omega\left(t^{*}\right)=\sup _{-\tau \leq t<+\infty} \omega(t)>0$. Obviously, if $t^{*}=-\tau$ then $\omega^{\prime}\left(t^{*}\right) \leq 0$, and if $t^{*} \in(-\tau, 0]$ then $\omega^{\prime}\left(t^{*}\right)=0$. However, from the boundary condition, we have $\omega^{\prime}\left(t^{*}\right)=\frac{1}{a} \omega\left(t^{*}\right)>0$, which gives a contraction. If $t^{*} \in(0,+\infty)$, then we have

$$
\omega\left(t^{*}\right)>0, \quad \omega^{\prime}\left(t^{*}\right)=0, \quad \omega^{\prime \prime}\left(t^{*}\right) \leq 0 .
$$


By the definition of auxiliary functions and $R>\sup _{t \in[0,+\infty)}\left|\beta^{\prime \prime \prime}(t)\right|$, we have

$$
\begin{aligned}
u^{(4)}\left(t^{*}\right)= & -q\left(t^{*}\right) F_{3}\left(t^{*},\left[u\left(t^{*}\right)\right],\left[u^{\prime}\left(t^{*}\right)\right],\left[u^{\prime \prime}\left(t^{*}\right)\right], u^{\prime \prime \prime}\left(t^{*}\right)\right) \\
= & -q\left(t^{*}\right) F_{2}\left(t^{*},\left[u\left(t^{*}\right)\right],\left[u^{\prime}\left(t^{*}\right)\right],\left[u^{\prime \prime}\left(t^{*}\right)\right], \beta^{\prime \prime \prime}\left(t^{*}\right)\right) \\
= & -q\left(t^{*}\right)\left[F_{1}\left(t^{*},\left[u\left(t^{*}\right)\right],\left[u^{\prime}\left(t^{*}\right)\right], \beta^{\prime \prime}\left(t^{*}\right), u^{\prime \prime}\left(t^{*}-\tau_{2,1}\left(t^{*}\right)\right), \ldots, \beta^{\prime \prime \prime}\left(t^{*}\right)\right)\right. \\
& \left.-\frac{u^{\prime \prime}\left(t^{*}\right)-\beta^{\prime \prime}\left(t^{*}\right)}{1+\left|u^{\prime \prime}\left(t^{*}\right)-\beta^{\prime \prime}\left(t^{*}\right)\right|}\right] .
\end{aligned}
$$

Now if $u^{\prime \prime}\left(t^{*}-\tau_{2,1}\left(t^{*}\right)\right)>\beta^{\prime \prime}\left(t^{*}-\tau_{2,1}\left(t^{*}\right)\right)$ from the definition of $\widetilde{z}_{1}$ it follows that

$$
\begin{aligned}
u^{(4)}\left(t^{*}\right)= & -q\left(t^{*}\right) F_{1}\left(t^{*},\left[u\left(t^{*}\right)\right],\left[u^{\prime}\left(t^{*}\right)\right], \beta^{\prime \prime}\left(t^{*}\right), \beta^{\prime \prime}\left(t^{*}-\tau_{2,1}\left(t^{*}\right)\right), u^{\prime \prime}\left(t^{*}-\tau_{2,2}\left(t^{*}\right)\right), \ldots,\right. \\
& \left.\beta^{\prime \prime \prime}\left(t^{*}\right)\right)+q\left(t^{*}\right) \frac{u^{\prime \prime}\left(t^{*}\right)-\beta^{\prime \prime}\left(t^{*}\right)}{1+\left|u^{\prime \prime}\left(t^{*}\right)-\beta^{\prime \prime}\left(t^{*}\right)\right|}
\end{aligned}
$$

and if $u^{\prime \prime}\left(t^{*}-\tau_{2,1}\left(t^{*}\right)\right) \leq \beta^{\prime \prime}\left(t^{*}-\tau_{2,1}\left(t^{*}\right)\right)$ from the condition $\left(\mathrm{H}_{5}\right)$, we have

$$
\begin{gathered}
u^{(4)}\left(t^{*}\right) \geq-q\left(t^{*}\right) F_{1}\left(t^{*},\left[u\left(t^{*}\right)\right],\left[u^{\prime}\left(t^{*}\right)\right], \beta^{\prime \prime}\left(t^{*}\right), \beta^{\prime \prime}\left(t^{*}-\tau_{2,1}\left(t^{*}\right)\right), u^{\prime \prime}\left(t^{*}-\tau_{2,2}\left(t^{*}\right)\right), \ldots,\right. \\
\left.\beta^{\prime \prime \prime}\left(t^{*}\right)\right)+q\left(t^{*}\right) \frac{u^{\prime \prime}\left(t^{*}\right)-\beta^{\prime \prime}\left(t^{*}\right)}{1+\left|u^{\prime \prime}\left(t^{*}\right)-\beta^{\prime \prime}\left(t^{*}\right)\right|}
\end{gathered}
$$

Similarly, we consider the cases $u^{\prime \prime}\left(t^{*}-\tau_{2, i}\left(t^{*}\right)\right)>\beta^{\prime \prime}\left(t^{*}-\tau_{2, i}\left(t^{*}\right)\right)$ or $u^{\prime \prime}\left(t^{*}-\tau_{2, i}\left(t^{*}\right)\right) \leq \beta^{\prime \prime}\left(t^{*}-\right.$ $\left.\tau_{2, i}\left(t^{*}\right)\right), i=2,3, \ldots, n$, and obtain the inequality

$$
u^{(4)}\left(t^{*}\right) \geq-q\left(t^{*}\right) F_{1}\left(t^{*},\left[u\left(t^{*}\right)\right],\left[u^{\prime}\left(t^{*}\right)\right],\left[\beta^{\prime \prime}\left(t^{*}\right)\right], \beta^{\prime \prime \prime}\left(t^{*}\right)\right)+q\left(t^{*}\right) \frac{u^{\prime \prime}\left(t^{*}\right)-\beta^{\prime \prime}\left(t^{*}\right)}{1+\left|u^{\prime \prime}\left(t^{*}\right)-\beta^{\prime \prime}\left(t^{*}\right)\right|}
$$

Next, if $u^{\prime}\left(t^{*}\right)>\beta^{\prime}\left(t^{*}\right)$ from the definition of $F_{1}$ it follows that

$$
\begin{aligned}
u^{(4)}\left(t^{*}\right) \geq & -q\left(t^{*}\right) F_{0}\left(t^{*},\left[u\left(t^{*}\right)\right], \beta^{\prime}\left(t^{*}\right), u^{\prime}\left(t^{*}-\tau_{1,1}\left(t^{*}\right)\right), \ldots,\left[\beta^{\prime \prime}\left(t^{*}\right)\right], \beta^{\prime \prime \prime}\left(t^{*}\right)\right) \\
& +q\left(t^{*}\right) \frac{u^{\prime \prime}\left(t^{*}\right)-\beta^{\prime \prime}\left(t^{*}\right)}{1+\left|u^{\prime \prime}\left(t^{*}\right)-\beta^{\prime \prime}\left(t^{*}\right)\right|}
\end{aligned}
$$

and if $u^{\prime}\left(t^{*}\right) \leq \beta^{\prime}\left(t^{*}\right)$ from the definition of $F_{1}$ and the condition $\left(\mathrm{H}_{4}\right)$ we have

$$
\begin{aligned}
u^{(4)}\left(t^{*}\right) \geq & -q\left(t^{*}\right) F_{0}\left(t^{*},\left[u\left(t^{*}\right)\right], \beta^{\prime}\left(t^{*}\right), u^{\prime}\left(t^{*}-\tau_{1,1}\left(t^{*}\right)\right), \ldots,\left[\beta^{\prime \prime}\left(t^{*}\right)\right], \beta^{\prime \prime \prime}\left(t^{*}\right)\right) \\
& +q\left(t^{*}\right) \frac{u^{\prime \prime}\left(t^{*}\right)-\beta^{\prime \prime}\left(t^{*}\right)}{1+\left|u^{\prime \prime}\left(t^{*}\right)-\beta^{\prime \prime}\left(t^{*}\right)\right|} .
\end{aligned}
$$

If $t^{*}-\tau_{1,1}\left(t^{*}\right)>0$, while discussing the cases $u^{\prime}\left(t^{*}-\tau_{1,1}\left(t^{*}\right)\right)>\beta^{\prime}\left(t^{*}-\tau_{1,1}\left(t^{*}\right)\right)$ we use the definition of $\widetilde{y}_{1}$, and when discussing the cases $u^{\prime}\left(t^{*}-\tau_{1,1}\left(t^{*}\right)\right) \leq \beta^{\prime}\left(t^{*}-\tau_{1,1}\left(t^{*}\right)\right)$ we use $\left(\mathrm{H}_{4}\right)$, and obtain

$$
\begin{aligned}
u^{(4)}\left(t^{*}\right) \geq & -q\left(t^{*}\right) F_{0}\left(t^{*},\left[u\left(t^{*}\right)\right], \beta^{\prime}\left(t^{*}\right), \beta^{\prime}\left(t^{*}-\tau_{1,1}\left(t^{*}\right)\right), \ldots,\left[\beta^{\prime \prime}\left(t^{*}\right)\right], \beta^{\prime \prime \prime}\left(t^{*}\right)\right) \\
& +q\left(t^{*}\right) \frac{u^{\prime \prime}\left(t^{*}\right)-\beta^{\prime \prime}\left(t^{*}\right)}{1+\left|u^{\prime \prime}\left(t^{*}\right)-\beta^{\prime \prime}\left(t^{*}\right)\right|}
\end{aligned}
$$


Similarly, if $t^{*}-\tau_{1,1}\left(t^{*}\right) \leq 0$, while discussing the cases $u^{\prime}\left(t^{*}-\tau_{1,1}\left(t^{*}\right)\right) \geq \beta^{\prime}\left(t^{*}-\tau_{1,1}\left(t^{*}\right)\right)$ we use $\left(\mathrm{H}_{4}\right)$, while when discussing the cases $u^{\prime}\left(t^{*}-\tau_{1,1}\left(t^{*}\right)\right)<\beta^{\prime}\left(t^{*}-\tau_{1,1}\left(t^{*}\right)\right)$ we use the definition of $\widetilde{y}_{1}$, to again find

$$
\begin{aligned}
u^{(4)}\left(t^{*}\right) \geq & -q\left(t^{*}\right) F_{0}\left(t^{*},\left[u\left(t^{*}\right)\right], \beta^{\prime}\left(t^{*}\right), \beta^{\prime}\left(t^{*}-\tau_{1,1}\left(t^{*}\right)\right), \ldots,\left[\beta^{\prime \prime}\left(t^{*}\right)\right], \beta^{\prime \prime \prime}\left(t^{*}\right)\right) \\
& +q\left(t^{*}\right) \frac{u^{\prime \prime}\left(t^{*}\right)-\beta^{\prime \prime}\left(t^{*}\right)}{1+\left|u^{\prime \prime}\left(t^{*}\right)-\beta^{\prime \prime}\left(t^{*}\right)\right|} .
\end{aligned}
$$

Following exactly as above, using the definition of $\widetilde{y}_{i}$ and $\left(\mathrm{H}_{4}\right)$, we consider the cases $i=$ $2, \ldots, n$ to finally obtain

$$
u^{(4)}\left(t^{*}\right) \geq-q\left(t^{*}\right) F_{0}\left(t^{*},\left[u\left(t^{*}\right)\right],\left[\beta^{\prime}\left(t^{*}\right)\right],\left[\beta^{\prime \prime}\left(t^{*}\right)\right], \beta^{\prime \prime \prime}\left(t^{*}\right)\right)+q\left(t^{*}\right) \frac{u^{\prime \prime}\left(t^{*}\right)-\beta^{\prime \prime}\left(t^{*}\right)}{1+\left|u^{\prime \prime}\left(t^{*}\right)-\beta^{\prime \prime}\left(t^{*}\right)\right|} .
$$

Next, if $u\left(t^{*}\right)>\beta\left(t^{*}\right)$ from the definition of $F_{0}$ it follows that

$$
\begin{aligned}
u^{(4)}\left(t^{*}\right) \geq & -q\left(t^{*}\right) f\left(t^{*}, \beta\left(t^{*}\right), u\left(t^{*}-\tau_{0,1}\left(t^{*}\right)\right), \ldots,\left[u\left(t^{*}\right)\right],\left[\beta^{\prime \prime}\left(t^{*}\right)\right], \beta^{\prime \prime \prime}\left(t^{*}\right)\right) \\
& +q\left(t^{*}\right) \frac{u^{\prime \prime}\left(t^{*}\right)-\beta^{\prime \prime}\left(t^{*}\right)}{1+\left|u^{\prime \prime}\left(t^{*}\right)-\beta^{\prime \prime}\left(t^{*}\right)\right|}
\end{aligned}
$$

and if $u\left(t^{*}\right) \leq \beta\left(t^{*}\right)$ from the definition of $F_{0}$ and the condition $\left(\mathrm{H}_{3}\right)$ we have

$$
\begin{aligned}
u^{(4)}\left(t^{*}\right) \geq & -q\left(t^{*}\right) f\left(t^{*}, \beta\left(t^{*}\right), u\left(t^{*}-\tau_{0,1}\left(t^{*}\right)\right), \ldots,\left[u\left(t^{*}\right)\right],\left[\beta^{\prime \prime}\left(t^{*}\right)\right], \beta^{\prime \prime \prime}\left(t^{*}\right)\right) \\
& +q\left(t^{*}\right) \frac{u^{\prime \prime}\left(t^{*}\right)-\beta^{\prime \prime}\left(t^{*}\right)}{1+\left|u^{\prime \prime}\left(t^{*}\right)-\beta^{\prime \prime}\left(t^{*}\right)\right|} .
\end{aligned}
$$

Similarly, we use the definition of $\widetilde{x}_{i}$ and $\left(\mathrm{H}_{3}\right)$ while discussing the cases $u\left(t^{*}-\tau_{0, i}\left(t^{*}\right)\right)>$ $\beta\left(t^{*}-\tau_{0, i}\left(t^{*}\right)\right)$ or $u\left(t^{*}-\tau_{0, i}\left(t^{*}\right)\right) \leq \beta\left(t^{*}-\tau_{0, i}\left(t^{*}\right)\right), i=1, \ldots, n$, to get

$$
u^{(4)}\left(t^{*}\right) \geq-q\left(t^{*}\right) f\left(t^{*},\left[\beta\left(t^{*}\right)\right],\left[\beta^{\prime}\left(t^{*}\right)\right],\left[\beta^{\prime \prime}\left(t^{*}\right)\right], \beta^{\prime \prime \prime}\left(t^{*}\right)\right)+q\left(t^{*}\right) \frac{u^{\prime \prime}\left(t^{*}\right)-\beta^{\prime \prime}\left(t^{*}\right)}{1+\left|u^{\prime \prime}\left(t^{*}\right)-\beta^{\prime \prime}\left(t^{*}\right)\right|},
$$

which implies that

$$
\omega^{\prime \prime}\left(t^{*}\right) \geq q\left(t^{*}\right) \frac{u^{\prime \prime}\left(t^{*}\right)-\beta^{\prime \prime}\left(t^{*}\right)}{1+\left|u^{\prime \prime}\left(t^{*}\right)-\beta^{\prime \prime}\left(t^{*}\right)\right|}>0,
$$

which is a contractions.

If $t^{*}=+\infty$ then $\omega(+\infty)=\sup _{t \in[-\tau,+\infty)} \omega(t)>0$. From the boundary conditions, we also have $\omega^{\prime}(+\infty)=u^{\prime \prime \prime}(+\infty)-\beta^{\prime \prime \prime}(+\infty) \leq 0$. But this implies that $\omega^{\prime \prime}(+\infty) \leq 0$ and $\omega^{\prime}(+\infty)=0$. However, now following as above, we find $\omega^{\prime \prime}(+\infty)>0$, which is a contradiction. Thus, $u^{\prime \prime}(t) \leq \beta^{\prime \prime}(t), t \in[-\tau,+\infty)$.

Consequently, we have

$$
\alpha^{\prime \prime}(t) \leq u^{\prime \prime}(t) \leq \beta^{\prime \prime}(t), \quad t \in[-\tau,+\infty)
$$

which on integration and using boundary conditions gives

$$
\beta^{\prime}(t) \leq u^{\prime}(t) \leq \alpha^{\prime}(t), \quad t \in[-\tau, 0), \quad \text { and } \quad \alpha^{\prime}(t) \leq u^{\prime}(t) \leq \beta^{\prime}(t), \quad t \in[0,+\infty)
$$


and now a further integration leads to

$$
\alpha(t) \leq u(t) \leq \beta(t), \quad t \in[-\tau,+\infty) .
$$

Further, since all conditions of the Lemma 3.1 are satisfied, $\|u\|_{\infty}^{3}<R$. Consequently, we have

$$
\begin{aligned}
u^{(4)}(t) & =-q(t) F_{3}\left(t,[u(t)],\left[u^{\prime}(t)\right],\left[u^{\prime \prime}(t)\right], u^{\prime \prime \prime}(t)\right) \\
& =-q(t) f\left(t,[u(t)],\left[u^{\prime}(t)\right],\left[u^{\prime \prime}(t)\right], u^{\prime \prime \prime}(t)\right)
\end{aligned}
$$

and hence, $u$ is a solution of (1.1)-(1.2).

Theorem 3.3 Assume that there exist two pairs of upper and lower solutions $\beta_{k}, \alpha_{k}, k=1,2$ of $B V P(1.1)-(1.2)$, where $\alpha_{2}, \beta_{1}$ are strict and

$$
\begin{aligned}
& \alpha_{2}^{(i)}(t) \not \leq \beta_{1}^{(i)}(t), \quad i=0,1,2, \\
& \alpha_{1}^{(i)}(t) \leq \alpha_{2}^{(i)}(t) \leq \beta_{2}^{(i)}(t), \quad \alpha_{1}^{(i)}(t) \leq \beta_{1}^{(i)}(t) \leq \beta_{2}^{(i)}(t), \quad t \in[-\tau,+\infty), i=0,2, \\
& \beta_{2}^{\prime}(t) \leq \alpha_{2}^{\prime}(t) \leq \alpha_{1}^{\prime}(t), \quad \beta_{2}^{\prime}(t) \leq \beta_{1}^{\prime}(t) \leq \alpha_{1}^{\prime}(t), \quad t \in[-\tau, 0), \\
& \alpha_{1}^{\prime}(t) \leq \alpha_{2}^{\prime}(t) \leq \beta_{2}^{\prime}(t), \quad \alpha_{1}^{\prime}(t) \leq \beta_{1}^{\prime}(t) \leq \beta_{2}^{\prime}(t), \quad t \in[0,+\infty),
\end{aligned}
$$

and $f$ satisfies Nagumo's condition with respect to $\alpha_{1}, \beta_{2}$. Suppose further that conditions $\left(\mathrm{H}_{2}\right)-\left(\mathrm{H}_{6}\right)$ hold with $\alpha$ and $\beta$ replaced by $\alpha_{1}$ and $\beta_{2}$, respectively. Then the problem (1.1)(1.2) has at least three solutions $u_{1}, u_{2}$, and $u_{3}$ such that

$$
\begin{aligned}
& \alpha_{k}^{\prime \prime}(t) \leq u_{k}^{\prime \prime}(t) \leq \beta_{k}^{\prime \prime}(t), \quad \alpha_{k}(t) \leq u_{k}(t) \leq \beta_{k}(t), \quad t \in[-\tau,+\infty), k=1,2, \\
& \beta_{k}^{\prime}(t) \leq u_{k}^{\prime}(t) \leq \alpha_{k}^{\prime}(t), \quad t \in[-\tau, 0), \quad \alpha_{k}^{\prime}(t) \leq u_{k}^{\prime}(t) \leq \beta_{k}^{\prime}(t), \quad t \in[0,+\infty), k=1,2, \\
& u_{3}^{(i)}(t) \not \leq \beta_{1}^{(i)}(t), \quad u_{3}^{(i)}(t) \nsupseteq \alpha_{2}^{(i)}(t), \quad t \in[-\tau,+\infty), i=0,1,2 .
\end{aligned}
$$

Proof First we define the truncated functions $\widetilde{F}_{0}, \widetilde{F}_{1}, \widetilde{F}_{2}, \widetilde{F}_{3}$ the same as $F_{0}, F_{1}, F_{2}, F_{3}$ in Theorem 3.2 with $\alpha, \beta$ replaced by $\alpha_{1}$ and $\beta_{2}$, respectively. Consider the modified differential equation

$$
u^{(4)}(t)+q(t) \widetilde{F}_{3}\left(t,[u(t)],\left[u^{\prime}(t)\right],\left[u^{\prime \prime}(t)\right], u^{\prime \prime \prime}(t)\right)=0, \quad 0 \leq t<+\infty .
$$

To show that (3.18)-(1.2) has at least three solutions, we define operators $\widetilde{T}_{2}, T_{2}$ as

$$
\widetilde{T}_{2} u(t)=\int_{0}^{\infty} G(t, s) q(s) \widetilde{F}_{3}\left(s,[u(s)],\left[u^{\prime}(s)\right],\left[u^{\prime \prime}(s)\right], u^{\prime \prime \prime}(s)\right) d s
$$

and

$$
T_{2} u(t)= \begin{cases}\psi_{2}(t), & -\tau \leq t \leq 0 \\ l(t)+\widetilde{T}_{2} u(t), & 0 \leq t<+\infty\end{cases}
$$


where $l(t)$ is as in (2.7) and

$$
\begin{aligned}
\psi_{2}(t)= & A+B t \\
& +\left(\theta(0)+a C+a \int_{0}^{\infty} q(s) \widetilde{F}_{3}\left(s,[u(s)],\left[u^{\prime}(s)\right],\left[u^{\prime \prime}(s)\right], u^{\prime \prime \prime}(s)\right) d s\right) \\
& \times\left(-a t-a^{2}+a^{2} e^{\frac{t}{a}}\right)+\int_{t}^{0}\left(s-a-t+a e^{\frac{t-s}{a}}\right) \theta(s) d s .
\end{aligned}
$$

As in Theorem 3.2, $T_{2}$ is completely continuous. By using the degree theory, we will show that $T_{2}$ has at least three fixed points which are solutions of (3.18)-(1.2). We note that $R$ in Lemma 3.1 instead of $\alpha, \beta$ now depends on $\alpha_{1}, \beta_{2}$. Set $\Omega_{2}=\{u \in X,\|u\|<N\}$ where $N$ is as in (3.15) then for any $u \in \bar{\Omega}_{2}$, it follows that $\left\|T_{2} u\right\|<N$, thus $T_{2} \bar{\Omega}_{2} \subset \Omega_{2}$, and so we have $\operatorname{deg}\left(I-T_{2}, \Omega_{2}, 0\right)=1$. Set

$$
\begin{aligned}
& \Omega_{\alpha_{2}}=\left\{u \in \Omega_{2}: u^{\prime \prime}(t)>\alpha_{2}^{\prime \prime}(t), t \in[-\tau,+\infty)\right\}, \\
& \Omega^{\beta_{1}}=\left\{u \in \Omega_{2}: u^{\prime \prime}(t)<\beta_{1}^{\prime \prime}(t), t \in[-\tau,+\infty)\right\} .
\end{aligned}
$$

Since $\alpha_{2}^{\prime \prime} \not \beta_{1}^{\prime \prime}, \alpha_{1}^{\prime \prime}(t) \leq \alpha_{2}^{\prime \prime}(t) \leq \beta_{2}^{\prime \prime}(t), \alpha_{1}^{\prime \prime}(t) \leq \beta_{1}^{\prime \prime}(t) \leq \beta_{2}^{\prime \prime}(t)$, we find $\Omega_{\alpha_{2}} \neq \emptyset \neq \Omega^{\beta_{1}}, \bar{\Omega}_{\alpha_{2}} \cap$ $\overline{\Omega^{\beta_{1}}}=\emptyset, \Omega_{2} \backslash \overline{\Omega_{\alpha_{2}} \cup \Omega^{\beta_{1}}} \neq \emptyset$. Now since $\alpha_{2}, \beta_{1}$ are strict lower and upper solutions there is no solution in $\partial \Omega_{\alpha_{2}} \cup \partial \Omega^{\beta_{1}}$. The additivity of degree implies that

$$
\begin{aligned}
\operatorname{deg}\left(I-T_{2}, \Omega_{2}, 0\right)= & \operatorname{deg}\left(I-T_{2}, \Omega_{2} \backslash \overline{\Omega_{\alpha_{2}} \cup \Omega^{\beta_{1}}}, 0\right) \\
& +\operatorname{deg}\left(I-T_{2}, \Omega_{\alpha_{2}}, 0\right)+\operatorname{deg}\left(I-T_{2}, \Omega^{\beta_{1}}, 0\right) .
\end{aligned}
$$

We will show that $\operatorname{deg}\left(I-T_{2}, \Omega_{\alpha_{2}}, 0\right)=\operatorname{deg}\left(I-T_{2}, \Omega^{\beta_{1}}, 0\right)=1$. For this, we define new operators $\widetilde{T}_{3}: \bar{\Omega}_{2} \rightarrow \bar{\Omega}_{2}$ and $T_{3}: \bar{\Omega}_{2} \rightarrow \bar{\Omega}_{2}$ as

$$
\widetilde{T}_{3} u(t)=\int_{0}^{\infty} q(s) \widehat{F}_{3}\left(s,[u(s)],\left[u^{\prime}(s)\right],\left[u^{\prime \prime}(s)\right], u^{\prime \prime \prime}(s)\right) d s
$$

and

$$
T_{3} u(t)= \begin{cases}\psi_{3}(t), & -\tau \leq t \leq 0 \\ l(t)+\widetilde{T}_{3} u(t), & 0 \leq t<+\infty\end{cases}
$$

where $l(t)$ is as in (2.7) and

$$
\begin{aligned}
\psi_{3}(t)= & A+B t \\
& +\left(\theta(0)+a C+a \int_{0}^{\infty} q(s) \widehat{F}_{3}\left(s,[u(s)],\left[u^{\prime}(s)\right],\left[u^{\prime \prime}(s)\right], u^{\prime \prime \prime}(s)\right) d s\right) \\
& \times\left(-a t-a^{2}+a^{2} e^{\frac{t}{a}}\right)+\int_{t}^{0}\left(s-a-t+a e^{\frac{t-s}{a}}\right) \theta(s) d s .
\end{aligned}
$$

Here the functions $\widehat{F}_{0}, \widehat{F}_{1}, \widehat{F}_{2}, \widehat{F}_{3}$ are same as $\widetilde{F}_{0}, \widetilde{F}_{1}, \widetilde{F}_{2}, \widetilde{F}_{3}$ except that $\alpha_{1}$ is replaced by $\alpha_{2}$. Now similar to the proof of Theorem 3.2 we find that $u$ is a fixed point of $T_{3}$ only 
when $\alpha_{2}^{\prime \prime}(t) \leq u^{\prime \prime}(t) \leq \beta_{2}^{\prime \prime}(t), t \in[-\tau,+\infty)$. Since the lower solution $\alpha_{2}$ is strict, $\alpha_{2}^{\prime \prime}(t) \neq u^{\prime \prime}(t)$, $t \in(-\tau,+\infty)$. Therefore, $u \in \Omega_{\alpha_{2}}$. Hence, it follows that

$$
\operatorname{deg}\left(I-T_{3}, \Omega_{2} \backslash \bar{\Omega}_{\alpha_{2}}, 0\right)=0 .
$$

Also, $T_{3} \bar{\Omega}_{2} \subset \Omega_{2}$, so that we have

$$
\operatorname{deg}\left(I-T_{3}, \Omega_{2}, 0\right)=1
$$

Therefore,

$$
\begin{aligned}
\operatorname{deg}\left(I-T_{2}, \Omega_{\alpha_{2}}, 0\right) & =\operatorname{deg}\left(I-T_{3}, \Omega_{\alpha_{2}}, 0\right) \\
& =\operatorname{deg}\left(I-T_{3}, \Omega_{\alpha_{2}}, 0\right)+\operatorname{deg}\left(I-T_{3}, \Omega_{2} \backslash \bar{\Omega}_{\alpha_{2}}, 0\right) \\
& =\operatorname{deg}\left(I-T_{3}, \Omega_{2}, 0\right)=1 .
\end{aligned}
$$

Similarly, we have

$$
\operatorname{deg}\left(I-T_{2}, \Omega^{\beta_{1}}, 0\right)=1
$$

and this leads to

$$
\operatorname{deg}\left(I-T_{2}, \Omega_{2} \backslash \overline{\Omega_{\alpha_{2}} \cup \Omega^{\beta_{1}}}, 0\right)=-1 .
$$

Finally, using the properties of the degree, we conclude that $T_{2}$ has at least three fixed points

$$
u_{1} \in \Omega_{\alpha_{2}}, \quad u_{2} \in \Omega^{\beta_{1}}, \quad u_{3} \in \Omega_{2} \backslash \overline{\Omega_{\alpha_{2}} \cup \Omega^{\beta_{1}}}
$$

which are the claimed solutions of the BVP (1.1)-(1.2).

\section{Examples}

Example 4.1 Consider the fourth-order nonlinear differential equation on the half-line with deviating arguments

$$
u^{(4)}(t)+\frac{1}{(1+t)^{3}} f\left(t,[u(t)],\left[u^{\prime}(t)\right],\left[u^{\prime \prime}(t)\right], u^{\prime \prime \prime}(t)\right)=0, \quad t \in(0,+\infty),
$$

where

$$
\begin{aligned}
f(t, & {\left.[u(t)],\left[u^{\prime}(t)\right],\left[u^{\prime \prime}(t)\right], u^{\prime \prime \prime}(t)\right) } \\
= & \frac{\left(u^{\prime \prime \prime}(t)-1\right)^{2}}{(1+t)^{4}}\left[\left(2 t+u^{\prime \prime}(t)\right)+\left(t+u^{\prime \prime}(t-1)\right)+\left(u^{\prime}(t / 3-1 / 3)-1\right)^{2}\right. \\
& \left.+\left(t^{3}+u(t / 2-1 / 2)\right)\right] .
\end{aligned}
$$

Clearly, (4.1) is a particular case of (1.1) with $q(t)=\frac{1}{(1+t)^{3}}$,

$$
[u(t)]=(u(t), u(t-t / 2-1 / 2)), \quad\left[u^{\prime}(t)\right]=\left(u^{\prime}(t), u^{\prime}(t-2 t / 3-1 / 3)\right),
$$




$$
\begin{aligned}
& {\left[u^{\prime \prime}(t)\right]=\left(u^{\prime \prime}(t), u^{\prime \prime}(t-1)\right),} \\
& \tau_{2,1}(t)=1, \quad \tau_{1,1}(t)=\frac{2 t}{3}+\frac{1}{3}, \quad \tau_{0,1}(t)=\frac{t}{2}+\frac{1}{2} .
\end{aligned}
$$

It follows that

$$
\tau=-\min _{0 \leq j \leq 2} \min \left(t-\tau_{j, 1}(t)\right)=1 .
$$

We consider (4.1) together with the following boundary conditions:

$$
\left\{\begin{array}{l}
u(0)=2, \quad u^{\prime}(0)=0, \quad u^{\prime \prime}(t)-\frac{1}{3} u^{\prime \prime \prime}(t)=\frac{4}{3} \quad \text { for } t \in[-1,0] \\
u^{\prime \prime \prime}(+\infty)=0 .
\end{array}\right.
$$

Comparing this with (1.2), we find $\theta(t)=\frac{4}{3}, a=\frac{1}{3}, A=2, B=0, C=0$.

For (4.1)-(4.2) a direct substitution shows that

$$
\beta(t)=\frac{t^{3}}{6}+\frac{4 t^{2}}{3}+\frac{11}{3}, \quad \alpha(t)=-\frac{t^{3}}{6}
$$

are upper and lower solutions such that $\beta, \alpha \in X \cap \mathcal{C}^{4}(0,+\infty)$. Further, for these functions we have

$$
\alpha^{\prime \prime}(t)=-t \leq \beta^{\prime \prime}(t)=t+\frac{8}{3}, \quad t \in[-1,+\infty) .
$$

We also note that when $t \in[0,+\infty)$ and

$$
\begin{aligned}
& \alpha(t / 2-1 / 2)=\frac{1}{48}-\frac{t}{16}+\frac{t^{2}}{16}-\frac{t^{3}}{48} \leq x_{1} \leq \beta(t / 2-1 / 2)=\frac{191}{48}-\frac{29 t}{48}+\frac{13 t^{2}}{48}+\frac{t^{3}}{48}, \\
& \beta^{\prime}(t / 3-1 / 3)=-\frac{5}{6}+\frac{7 t}{9}+\frac{t^{2}}{18} \leq y_{1} \leq \alpha^{\prime}(t / 3-1 / 3)=-\frac{1}{18}+\frac{t}{9}-\frac{t^{2}}{18}, \quad t \in[0,1), \\
& \alpha^{\prime}(t / 3-1 / 3)=-\frac{1}{18}+\frac{t}{9}-\frac{t^{2}}{18} \leq y_{1} \leq \beta^{\prime}(t / 3-1 / 3)=-\frac{5}{6}+\frac{7 t}{9}+\frac{t^{2}}{18}, \quad t \in[1,+\infty), \\
& \alpha^{\prime \prime}(t)=-t \leq z_{0} \leq \beta^{\prime \prime}(t)=t+\frac{8}{3}, \quad \alpha^{\prime \prime}(t-1)=-t+1 \leq z_{1} \leq \beta^{\prime \prime}(t-1)=t+\frac{5}{3},
\end{aligned}
$$

the function $f$ is continuous and satisfies Nagumo's condition with respect to $\alpha$ and $\beta$, that is,

$$
\begin{aligned}
& \left|f\left(t, x_{0}, x_{1}, y_{0}, y_{1}, z_{0}, z_{1}, w\right)\right| \\
& \quad=\left|(w-1)^{2} \frac{\left(2 t+z_{0}\right)+\left(t+z_{1}\right)+\left(y_{1}-1\right)^{2}+\left(t^{3}+x_{1}\right)}{(1+t)^{4}}\right| \\
& \quad \leq\left(\sup _{t \in[0,+\infty)} \frac{\frac{t^{4}}{324}+\frac{1,435 t^{3}}{1,296}+\frac{871 t^{2}}{1,296}+\frac{5,393 t}{1,296}+\frac{1,681}{144}}{(1+t)^{4}}\right)(|w|+1)^{2} \\
& \quad \leq 12(|w|+1)^{2} .
\end{aligned}
$$

Hence we can take $\varphi(t)=12$ and $h(w)=(w+1)^{2}$. Now if $1<\gamma \leq 3$, then

$$
\sup _{t \in[0,+\infty)}(1+t)^{\gamma} \frac{12}{(1+t)^{3}}=\sup _{t \in[0,+\infty)} \frac{12}{(1+t)^{3-\gamma}} \leq 12<+\infty
$$


and

$$
\begin{aligned}
& \int_{0}^{\infty} \frac{1}{(1+s)^{3}} d s<+\infty, \quad \int_{0}^{\infty} \frac{s}{(1+s)^{3}} d s<+\infty \\
& \int_{0}^{\infty} \frac{s}{h(s)} d s=\int_{0}^{\infty} \frac{s}{(s+1)^{2}} d s=+\infty
\end{aligned}
$$

and these imply that conditions $\left(\mathrm{H}_{1}\right),\left(\mathrm{H}_{2}\right)$, and $\left(\mathrm{H}_{6}\right)$ are fulfilled. Now we will show that $f$ satisfies conditions $\left(\mathrm{H}_{3}\right)-\left(\mathrm{H}_{5}\right)$ of Theorem 3.2. For $t \in[0,+\infty), y_{i}, z_{i}, w \in \mathbb{R}, i=0,1$, when

$$
\alpha\left(t-\tau_{0,1}(t)\right)=\alpha(t / 2-1 / 2) \leq x_{1} \leq \beta(t / 2-1 / 2)=\beta\left(t-\tau_{0,1}(t)\right)
$$

since $f$ is increasing with respect to $x_{1}$,

$$
\begin{aligned}
f\left(t, x_{0}, \alpha(t / 2-1 / 2), y_{0}, y_{1}, z_{0}, z_{1}, w\right) & \leq f\left(t, x_{0}, x_{1}, y_{0}, y_{1}, z_{0}, z_{1}, w\right) \\
& \leq f\left(t, x_{0}, \beta(t / 2-1 / 2), y_{0}, y_{1}, z_{0}, z_{1}, w\right),
\end{aligned}
$$

for $x_{i}, z_{i}, w \in \mathbb{R}, i=0,1$, when

$$
\begin{aligned}
& \beta^{\prime}\left(t-\tau_{1,1}(t)\right)=\beta^{\prime}(t / 3-1 / 3) \leq y_{1} \leq \alpha^{\prime}\left(t-\tau_{1,1}(t)\right)=\alpha^{\prime}(t / 3-1 / 3), \\
& \quad \text { if } t-\tau_{1,1} \leq 0, t \in[0,1),
\end{aligned}
$$

or

$$
\begin{aligned}
& \alpha^{\prime}\left(t-\tau_{1,1}(t)\right)=\alpha^{\prime}(t / 3-1 / 3) \leq y_{1} \leq \beta^{\prime}\left(t-\tau_{0,1}(t)\right)=\beta^{\prime}(t / 3-1 / 3), \\
& \quad \text { if } t-\tau_{1,1}>0, t \in[1,+\infty),
\end{aligned}
$$

since $f$ is decreasing on $\left[\beta^{\prime}\left(t-\tau_{1,1}\right), \alpha^{\prime}\left(t-\tau_{1,1}(t)\right)\right]$ for $t \in[0,1)$ and increasing on $\left[\alpha^{\prime}(t-\right.$ $\left.\left.\tau_{1,1}(t)\right), \beta^{\prime}\left(t-\tau_{1,1}\right)\right]$ for $t \in[1,+\infty)$ with respect to $y_{1}$,

$$
\begin{aligned}
f\left(t, x_{0}, x_{1}, y_{0}, \alpha^{\prime}(t / 3-1 / 3), z_{0}, z_{1}, w\right) & \leq f\left(t, x_{0}, x_{1}, y_{0}, y_{1}, z_{0}, z_{1}, w\right) \\
& \leq f\left(t, x_{0}, x_{1}, y_{0}, \beta^{\prime}(t / 3-1 / 3), z_{0}, z_{1}, w\right)
\end{aligned}
$$

and for $t \in[0,+\infty), x_{i}, y_{i}, w \in \mathbb{R}, i=0,1$, when

$$
\alpha^{\prime \prime}(t) \leq z_{0} \leq \beta^{\prime \prime}(t)
$$

since $f$ is increasing with respect to $z_{0}$,

$$
f\left(t, x_{0}, x_{1}, y_{0}, y_{1}, \alpha^{\prime \prime}(t), z_{1}, w\right) \leq f\left(t, x_{0}, x_{1}, y_{0}, y_{1}, z_{0}, z_{1}, w\right) \leq f\left(t, x_{0}, x_{1}, y_{0}, y_{1}, \beta^{\prime \prime}(t), z_{1}, w\right)
$$

also when

$$
\alpha^{\prime \prime}\left(t-\tau_{2,1}(t)\right)=\alpha^{\prime \prime}(t-1) \leq z_{1} \leq \beta^{\prime}\left(t-\tau_{2,1}(t)\right)=\beta^{\prime \prime}(t-1)
$$


since $f$ is increasing respect to $z_{1}$,

$$
\begin{aligned}
f\left(t, x_{0}, x_{1}, y_{0}, y_{1}, z_{0}, \alpha^{\prime \prime}(t-1), w\right) & \leq f\left(t, x_{0}, x_{1}, y_{0}, y_{1}, z_{0}, z_{1}, w\right) \\
& \leq f\left(t, x_{0}, x_{1}, y_{0}, y_{1}, z_{0}, \beta^{\prime \prime}(t-1), w\right) .
\end{aligned}
$$

Theorem 3.2 now ensures that the BVP (4.1)-(4.2) has at least one solution $u(t)$ such that

$$
-\frac{t^{3}}{6} \leq u(t) \leq \frac{t^{3}}{6}+\frac{4 t^{2}}{3}+\frac{11}{3}, \quad-t \leq u^{\prime \prime}(t) \leq t, \text { for all } t \in[-1,+\infty),
$$

and

$$
\begin{aligned}
& \frac{t^{2}}{2}+\frac{8 t}{3} \leq u^{\prime}(t) \leq-\frac{t^{2}}{2}, \quad \text { for all } t \in[-1,0), \\
& -\frac{t^{2}}{2} \leq u^{\prime}(t) \leq \frac{t^{2}}{2}+\frac{8 t}{3}, \quad \text { for all } t \in[0,+\infty),
\end{aligned}
$$

also $\|u\|_{\infty}^{3}<R$ where $R>\sqrt{\exp (192)\left(1+\eta^{2}\right)}, \eta \geq 4$, and $\gamma=2$.

Example 4.2 Consider the fourth-order nonlinear differential equation on the half-line with deviating arguments

$$
u^{(4)}(t)+\frac{1}{(1+t)^{3}} f\left(t,[u(t)],\left[u^{\prime}(t)\right],\left[u^{\prime \prime}(t)\right], u^{\prime \prime \prime}(t)\right)=0, \quad t \in(0,+\infty),
$$

where

$$
\begin{aligned}
f\left(t,[u(t)],\left[u^{\prime}(t)\right],\left[u^{\prime \prime}(t)\right], u^{\prime \prime \prime}(t)\right) \\
=\left(\frac{23}{28}-u^{\prime \prime \prime}(t)\right) \\
\quad+\frac{\left(1-\left(u^{\prime \prime \prime}(t)\right)^{2}\right)^{2}\left(\frac{9}{16}-\left(u^{\prime \prime \prime}(t)\right)^{2}\right)^{2}\left(\frac{6}{7}-u^{\prime \prime \prime}(t)\right)^{2}\left[\left(1+u^{\prime \prime}(t-1)\right)+\left(u^{\prime}\left(t-\frac{1}{2}\right)-\frac{1}{2}\right)^{2}+\left(u\left(t-\frac{1}{3}\right)+1\right)\right]}{\left(u^{\prime \prime \prime}(t)^{2}+1\right)^{4}(1+t)^{4}} .
\end{aligned}
$$

Clearly, (4.3) is a particular case of (1.1) with $q(t)=\frac{1}{(1+t)^{3}}$,

$$
\begin{aligned}
& {[u(t)]=(u(t), u(t-1 / 3)), \quad\left[u^{\prime}(t)\right]=\left(u^{\prime}(t), u^{\prime}(t-1 / 2)\right),} \\
& {\left[u^{\prime \prime}(t)\right]=\left(u^{\prime \prime}(t), u^{\prime \prime}(t-1)\right),} \\
& \tau_{2,1}(t)=1, \quad \tau_{1,1}(t)=1 / 2, \quad \tau_{0,1}(t)=1 / 3 .
\end{aligned}
$$

It follows that

$$
\tau=-\min _{0 \leq j \leq 2} \min _{t \geq 0}\left(t-\tau_{j, 1}\right)=1 .
$$

We consider (4.3) together with the following boundary conditions:

$$
\left\{\begin{array}{l}
u(0)=\frac{13}{2}, \quad u^{\prime}(0)=4, \quad u^{\prime \prime}(t)-2 u^{\prime \prime \prime}(t)=t-1 \quad t \in[-1,0] \\
u^{\prime \prime \prime}(+\infty)=\frac{23}{28} .
\end{array}\right.
$$

Comparing this with (1.2), we find $\theta(t)=t-1, a=\frac{1}{3}, A=\frac{13}{2}, B=4, C=\frac{23}{28}$. 
For (4.3)-(4.4) we take $\alpha_{1}, \alpha_{2}$ and $\beta_{1}, \beta_{2}$ as follows:

$$
\alpha_{1}(t)=-\frac{t^{3}}{6}-2 t^{2}+4 t, \quad \alpha_{2}(t)=\frac{t^{3}}{8}+t^{2}+4 t+6
$$

and

$$
\beta_{1}(t)=\frac{t^{3}}{7}+4 t+7, \quad \beta_{2}(t)=\frac{t^{3}}{6}+2 t^{2}+4 t+7
$$

and by direct substitution verify that $\alpha_{2}, \beta_{1} \in X \cap \mathcal{C}^{4}(0,+\infty)$ are its strict lower and upper solutions, and $\alpha_{1}, \beta_{2} \in X \cap \mathcal{C}^{4}(0,+\infty)$ are lower and upper solutions, and satisfy the assumption (3.17).

We also verify that for every $t \in[0,+\infty), w \in \mathbb{R}$,

$$
\begin{aligned}
& -t-3 \leq z_{1} \leq t+3, \quad-\frac{t^{3}}{6}-\frac{11 t^{2}}{6}+\frac{95 t}{18}-\frac{251}{162} \leq x_{1} \leq \frac{t^{3}}{6}+\frac{11 t^{2}}{6}+\frac{49 t}{18}+\frac{953}{162}, \\
& \frac{t^{2}}{2}+\frac{7 t}{2}+\frac{17}{8} \leq y_{1} \leq-\frac{t^{2}}{2}-\frac{7 t}{2}+\frac{47}{8}, \quad t \in[0,1 / 2) \quad \text { and } \\
& -\frac{t^{2}}{2}-\frac{7 t}{2}+\frac{47}{8} \leq y_{1} \leq \frac{t^{2}}{2}+\frac{7 t}{2}+\frac{17}{8}, \quad t \in[1 / 2,+\infty),
\end{aligned}
$$

we have

$$
\begin{aligned}
\mid f & \left.t, x_{0}, x_{1}, y_{0}, y_{1}, z_{0}, z_{1}, w\right) \mid \\
& =\left|(23 / 28-w)+\frac{\left(1-w^{2}\right)^{2}\left(\frac{9}{16}-w^{2}\right)^{2}\left(\frac{6}{7}-w\right)^{2}\left[\left(1+z_{1}\right)+\left(y_{1}-\frac{1}{2}\right)^{2}+\left(x_{1}+1\right)\right]}{\left(w^{2}+1\right)^{4}(1+t)^{4}}\right| \\
& \left.\leq(1+|w|)+(1+|w|)^{2} \frac{\frac{206,185}{5,184}+\frac{1,087 t}{72}+\frac{377 t^{2}}{24}+\frac{11 t^{3}}{3}+\frac{t^{4}}{4}}{(1+t)^{4}}\right] \\
& \leq(1+|w|)^{2}\left[1+\sup _{t \in[0,+\infty)} \frac{\frac{206,185}{5,184}+\frac{1,087 t}{72}+\frac{377 t^{2}}{24}+\frac{11 t^{3}}{3}+\frac{t^{4}}{4}}{(1+t)^{4}}\right] \\
& \leq 41(1+|w|)^{2}=\varphi(t) h(|w|) .
\end{aligned}
$$

Hence the function $f$ satisfies Nagumo's condition with $h(w)=(w+1)^{2}$ and $\varphi(t)=41$. Now if $1<\gamma \leq 3$, then

$$
\sup _{t \in[0,+\infty)}(1+t)^{\gamma} \frac{41}{(1+t)^{3}}=\sup _{t \in[0,+\infty)} \frac{41}{(1+t)^{3-\gamma}} \leq 41<+\infty
$$

and

$$
\begin{aligned}
& \int_{0}^{\infty} \frac{1}{(1+s)^{3}} d s<+\infty, \quad \int_{0}^{\infty} \frac{s}{(1+s)^{3}} d s<+\infty \\
& \int_{0}^{\infty} \frac{s}{h(s)} d s=\int_{0}^{\infty} \frac{s}{(s+1)^{2}} d s=+\infty
\end{aligned}
$$


and these imply that conditions $\left(\mathrm{H}_{1}\right),\left(\mathrm{H}_{2}\right)$, and $\left(\mathrm{H}_{6}\right)$ are fulfilled. Now we shall show that $f$ satisfies conditions $\left(\mathrm{H}_{3}\right)-\left(\mathrm{H}_{5}\right)$ of Theorem 3.2. For $t \in[0,+\infty), y_{i}, z_{i}, w \in \mathbb{R}, i=0,1$, when

$$
\alpha\left(t-\tau_{0,1}(t)\right)=\alpha(t-1 / 3) \leq x_{1} \leq \beta(t-1 / 3)=\beta\left(t-\tau_{0,1}(t)\right),
$$

since $f$ is increasing with respect to $x_{1}$,

$$
\begin{aligned}
f\left(t, x_{0}, \alpha(t-1 / 3), y_{0}, y_{1}, z_{0}, z_{1}, w\right) & \leq f\left(t, x_{0}, x_{1}, y_{0}, y_{1}, z_{0}, z_{1}, w\right) \\
& \leq f\left(t, x_{0}, \beta(t-1 / 3), y_{0}, y_{1}, z_{0}, z_{1}, w\right)
\end{aligned}
$$

for $x_{i}, z_{i}, w \in \mathbb{R}, i=0,1$, when

$$
\begin{aligned}
& \beta^{\prime}\left(t-\tau_{1,1}(t)\right)=\beta^{\prime}(t-1 / 2) \leq y_{1} \leq \alpha^{\prime}\left(t-\tau_{1,1}(t)\right)=\alpha^{\prime}(t-1 / 2), \\
& \quad \text { if } t-\tau_{1,1} \leq 0, t \in[0,1 / 2),
\end{aligned}
$$

or

$$
\begin{aligned}
& \alpha^{\prime}\left(t-\tau_{1,1}(t)\right)=\alpha^{\prime}(t-1 / 2) \leq y_{1} \leq \beta^{\prime}\left(t-\tau_{0,1}(t)\right)=\beta^{\prime}(t-1 / 2), \\
& \quad \text { if } t-\tau_{1,1}>0, t \in[1 / 2,+\infty) .
\end{aligned}
$$

Since $f$ is decreasing on $\left[\beta^{\prime}\left(t-\tau_{1,1}\right), \alpha^{\prime}\left(t-\tau_{1,1}(t)\right)\right]$ for $t \in[0,1 / 2)$ and increasing on $\left[\alpha^{\prime}(t-\right.$ $\left.\left.\tau_{1,1}(t)\right), \beta^{\prime}\left(t-\tau_{1,1}\right)\right]$ for $t \in[1 / 2,+\infty)$ with respect to $y_{1}$,

$$
\begin{aligned}
f\left(t, x_{0}, x_{1}, y_{0}, \alpha^{\prime}(t-1 / 2), z_{0}, z_{1}, w\right) & \leq f\left(t, x_{0}, x_{1}, y_{0}, y_{1}, z_{0}, z_{1}, w\right) \\
& \leq f\left(t, x_{0}, x_{1}, y_{0}, \beta^{\prime}(t-1 / 2), z_{0}, z_{1}, w\right)
\end{aligned}
$$

and for $t \in[0,+\infty), x_{i}, y_{i}, w \in \mathbb{R}, i=0,1$, when

$$
\alpha^{\prime \prime}\left(t-\tau_{2,1}(t)\right)=\alpha^{\prime \prime}(t-1) \leq z_{1} \leq \beta^{\prime}\left(t-\tau_{2,1}(t)\right)=\beta^{\prime \prime}(t-1)
$$

since $f$ is increasing with respect to $z_{1}$,

$$
\begin{aligned}
f\left(t, x_{0}, x_{1}, y_{0}, y_{1}, z_{0}, \alpha^{\prime \prime}(t-1), w\right) & \leq f\left(t, x_{0}, x_{1}, y_{0}, y_{1}, z_{0}, z_{1}, w\right) \\
& \leq f\left(t, x_{0}, x_{1}, y_{0}, y_{1}, z_{0}, \beta^{\prime \prime}(t-1), w\right) .
\end{aligned}
$$

This ensures that in Theorem 3.3 all assumptions $\left(\mathrm{H}_{1}\right)-\left(\mathrm{H}_{6}\right)$ are fulfilled. Therefore, we conclude that the problem (4.3)-(4.4) has at least three solutions. 


\section{Author details}

'Economics, Commercial and Management Sciences, Preparatory School of Oran, BP 65 CH 2 Achaba Hnifi, Technople de I'USTO, Bir El Djir, Algeria. ${ }^{2}$ Department of Mathematics, Texas A\&M University-Kingsville, 700 University Blvd., Kingsville, TX 78363-8202, USA. ${ }^{3}$ Department of Mathematics, Faculty of Science, King Abdulaziz University, P.O. Box 80203, Jeddah, 21589, Saudi Arabia. ${ }^{4}$ Department of Mathematics, Faculty of Science, Ege University, Bornova, Izmir, 35100, Turkey. ${ }^{5}$ Mathematics Faculty of Science, Oran University, Es-Senia, BP1524, Algeria.

\section{Acknowledgements}

The authors are grateful for the referees' careful reading and comments on this paper that led to the improvement of the original manuscript. This work was done when the third author was on academic leave, visiting Texas A\&M University Kingsville, Department of Mathematics. He gratefully acknowledges the financial support of The Scientific and Technological Research Council of Turkey (TUBITAK).

Received: 20 February 2015 Accepted: 22 May 2015 Published online: 24 June 2015

\section{References}

1. Agarwal, RP: Boundary value problems for differential equations with deviating arguments. J. Math. Phys. Sci. 6, 425-438 (1972)

2. Bai, C, Fang, J: On positive solutions of boundary value problems for second-order functional differential equations on infinite intervals. J. Math. Anal. Appl. 282, 711-731 (2003)

3. Eloe, PW, Grimm, LJ: Conjugate type boundary value problems for functional differential equations. Rocky Mt. J. Math. $12,627-633(1982)$

4. Erbe, LH, Kong, Q: Boundary value problems for singular second-order functional differential equations. J. Comput. Appl. Math. 53, 377-388 (1994)

5. Figueroa, R, Pouso, RL: Minimal and maximal solutions to first-order differential equations with state-dependent deviated arguments. Bound. Value Probl. 2012, Article ID 7 (2012). doi:10.1186/1687-2770-2012-7

6. Graef, JR, Kong, L, Minhós, FM, Fialho, J: On the lower and upper solution method for higher order functional boundary value problems. Appl. Anal. Discrete Math. 5, 133-146 (2011)

7. Grimm, LJ, Schmitt, K: Boundary value problems for delay-differential equations. Bull. Am. Math. Soc. 74, 997-1000 (1968)

8. Grimm, LJ, Schmitt, K: Boundary value problems for differential equations with deviating arguments. Aequ. Math. 4, $176-190(1970)$

9. Hale, JK, Verduyn Lunel, SM: Introduction to Functional Differential Equations. Applied Mathematical Sciences, vol. 99 Springer, New York (1993)

10. Henderson, J: Boundary Value Problem for Functional Differential Equations. World Scientific, Singapore (1995)

11. Jiang, D, Yang, Y, Chu, J, O'Regan, D: The monotone method for Neumann functional differential equations with upper and lower solutions in the reverse order. Nonlinear Anal., Theory Methods Appl. 67, 2815-2828 (2007)

12. Kamenskii, GA: Extrema of Nonlocal Functionals and Boundary Value Problems for Functional Differential Equations. Nova Science Publishers, New York (2007)

13. Lian, H, Agarwal, RP, Song, J: Boundary value problems for differential equations with deviating arguments. (submitted)

14. Philos, CG: Positive solutions to a higher-order nonlinear delay boundary value problem on the half line. Bull. Lond. Math. Soc. 41, 872-884 (2009)

15. Weng, P: Boundary value problems for second-order mixed-type functional differential equations. Appl. Math. J. Chin Univ. Ser B 12, 155-164 (1997)

16. Wei, Y: Existence and uniqueness of solutions for a second-order delay differential equation boundary value problem on the half-line. Bound. Value Probl. (2008). doi:10.1155/2008/752827

17. Philos, CG: Positive increasing solutions on the half line to second order nonlinear delay differential equations. Glasg. Math. J. 49, 197-211 (2007)

18. Agarwal, RP, O'Regan, D: Infinite Interval Problems for Differential, Difference and Integral Equations. Kluwer Academic, Dordrecht (2001)

19. Agarwal, RP, O'Regan, D: Nonlinear boundary value problems on the semi-infinite interval: an upper and lower solution approach. Mathematika 49, 129-140 (2002)

20. Bai, C, Li, C: Unbounded upper and lower solution method for third-order boundary-value problems on the half-line. Electron. J. Differ. Equ. 2009, 119 (2009)

21. Lian, H, Wang, P, Ge, W: Unbounded upper and lower solutions method for Sturm-Liouville boundary value problem on infinite intervals. Nonlinear Anal., Theory Methods Appl. 70, 2627-2633 (2009)

22. Lian, $\mathrm{H}$, Zhao, J: Existence of unbounded solutions for a third-order boundary value problem on infinite intervals. Discrete Dyn. Nat. Soc. 2012, Article ID 357697 (2012). doi:10.1155/2012/357697

23. Lian, H, Zhao, J, Agarwal, RP: Upper and lower solution method for $n$ th-order BVPs on an infinite interval. Bound. Value Probl. 2014, Article ID 100 (2014). doi:10.1186/1687-2770-2014-100

24. Yan, B, O'Regan, D, Agarwal, RP: Unbounded solutions for singular boundary value problems on the semi-infinite interval: upper and lower solutions and multiplicity. J. Comput. Appl. Math. 197, 365-386 (2006)

25. Du, Z, Liu, W, Lin, X: Multiple solutions to a three-point boundary value problem for higher-order ordinary differential equations. J. Math. Anal. Appl. 335, 1207-1218 (2007)

26. Ehme, J, Eloe, PW, Henderson, J: Upper and lower solution methods for fully nonlinear boundary value problems. J. Differ. Equ. 180, 51-64 (2002)

27. Minhós, F, Gyulov, T, Santos, Al: Existence and location result for a fourth-order boundary value problem. Discrete Contin. Dyn. Syst. 2005, 662-671 (2005)

28. Eloe, PW, Kaufmann, ER, Tisdell, CC: Multiple solutions of a boundary value problem on an unbounded domain. Dyn. Syst. Appl. 15(1), 53-63 (2006) 
29. Zhao, $Y, C h e n, H, X u, C$ : Existence of multiple solutions for three-point boundary-value problems on infinite intervals in Banach spaces. Electron. J. Differ. Equ. 2012, 44 (2012)

30. Graef, JR, Kong, L, Minhós, FM: Higher order $\phi$-Laplacian BVP with generalized Sturm-Liouville boundary conditions. Differ. Equ. Dyn. Syst. 18(4), 373-383 (2010)

Submit your manuscript to a SpringerOpen ${ }^{\circ}$ journal and benefit from:

- Convenient online submission

Rigorous peer review

- Immediate publication on acceptance

Open access: articles freely available online

- High visibility within the field

- Retaining the copyright to your article

Submit your next manuscript at $\boldsymbol{~ s p r i n g e r o p e n . c o m ~}$ 